\title{
The geometrical theory of constraints applied to the dynamics of vakonomic mechanical systems: The vakonomic bracket
}

\author{
Sonia Martínez, ${ }^{\text {a) }}$ Jorge Cortés, ${ }^{\text {b) }}$ and Manuel de León ${ }^{c)}$ \\ Instituto de Matemáticas y Física Fundamental, Consejo Superior de Investigaciones \\ Científicas, Serrano 123, 28006 Madrid, Spain
}

(Received 2 August 1999; accepted for publication 14 September 1999)

A vakonomic mechanical system can be alternatively described by an extended Lagrangian using the Lagrange multipliers as new variables. Since this extended Lagrangian is singular, the constraint algorithm can be applied and a Dirac bracket giving the evolution of the observables can be constructed. (C) 2000 American Institute of Physics. [S0022-2488(00)02802-4]

\section{INTRODUCTION}

There are two different approaches to Lagrangian systems subjected to nonholonomic constraints. The first one is based on the d'Alembert principle ${ }^{1-5}$ and the corresponding equations of motion are termed nonholonomic. The second approach is purely variational and was proposed by Kozloz. ${ }^{6}$ Arnold, Kozlov, and Neishtadt ${ }^{1}$ coined the name of vakonomic (mechanics of variational axiomatic kind) to refer to that sort of mechanics. Interesting comparisons between both approaches can be found in Refs. 3, 7, and 8 .

Both topics have received a lot of attention in recent years in the context of geometric mechanics. Nonholonomic mechanics has been studied from a Hamiltonian point of view, ${ }^{9-11}$ from a Lagrangian one, ${ }^{12-17}$ and even from a Poisson one. ${ }^{18-20}$ Several papers are devoted to highlighting the equivalence among these viewpoints. ${ }^{21-23}$ Indeed, nonholonomic mechanics has many applications to engineering (robotics, control of satellites, etc.), since it seems appropriate to model the dynamical behavior of phenomena like rolling, etc. (see Ref. 2, and references therein). On the other hand, vakonomic mechanics is applied to study problems of optimal control theory (being related to sub-Riemannian geometry), ${ }^{24,25}$ economic growth theory, ${ }^{26}$ motion of microorganisms at low Reynolds number, ${ }^{27}$ etc. A geometric unified approach was recently developed in Ref. 28.

The aim of this paper is to study the equations of motion of vakonomic mechanical systems in the framework of singular Lagrangian theories. As is well known, a vakonomic system given by a Lagrangian function $L=L\left(q^{A}, \dot{q}^{A}\right)$ and constraints $\Phi_{i}\left(q^{A}, \dot{q}^{A}\right)=0$, can be equivalently described by the extended Lagrangian $\mathcal{L}=\mathcal{L}\left(q^{A}, \lambda^{i}, \dot{q}^{A}, \dot{\lambda}^{i}\right)=L\left(q^{A}, \dot{q}^{A}\right)+\lambda^{i} \Phi_{i}$ (see Ref. 1). This new Lagrangian is obviously singular, and its dynamics can be studied using Dirac's machinery of constraints. ${ }^{29}$ A first step in this direction is due to Cariñena and Rañada, ${ }^{30}$ where they considered a global constraint function and treated the problem in the Lagrangian formalism.

Our program here is to apply the geometric version of the Dirac-Bergmann constraint algorithm due to Gotay and Nester ${ }^{31-33}$ to the extended Lagrangian $\mathcal{L}$. For that purpose, we first enlarge the original space of velocities $Q$ to $P=Q \times \mathbb{R}^{m}$, and then we apply Gotay-Nester's procedure to $\mathcal{L}$. We assume that $L$ is a natural Lagrangian, that is, $L=T-U$ where $T$ is the kinetic energy derived from a Riemannian metric on $Q$, and $U$ is the potential energy. In addition, the constraints are supposed to be linear in the velocities. With these assumptions, we find that the algorithm stabilizes at the second step or, in other words, there are only secondary constraints.

\footnotetext{
${ }^{a)}$ Electronic mail: ceem304@imaff.cfmac.csic.es

${ }^{b)}$ Electronic mail: ceec306@imaff.cfmac.csic.es

${ }^{c)}$ Electronic mail: mdeleon@imaff.cfmac.csic.es
} 
Moreover, all the constraints are second class in according with Dirac's terminology. This last fact implies that the final constraint submanifold $M_{2}$ is symplectic with respect to the canonical symplectic structure on $T^{*} P$ and the symplectic structure induced there provides a Poisson bracket that is just the same induced by the ambient Dirac bracket. ${ }^{29,34}$ A first result is that this procedure "reduces" the phase space from $T^{*} P$ to $M_{2}$.

Furthermore, the final constraint submanifold is diffeomorphic with $\bar{M} \times \mathrm{R}^{m}$, where $\bar{M}$ is the image in $T^{*} Q$ by the Legendre transformation of $M$. An interesting consequence of this identification is the possibility of defining a Poisson bracket on functions on $\bar{M}$ which produces a function on $M_{2}$ (since we have to take account of the Lagrange multipliers). We are then impelled to call this bracket the vakonomic bracket, in distinction with the so-called nonholonomic bracket in nonholonomic mechanics. ${ }^{19,20,21,23,35}$ Indeed, the vakonomic bracket gives the evolution of the observables of the vakonomic system.

If we consider a more general kind of constraints or Lagrangian not necessarily regular (situations which are more common in applications), the process is of course very much involved, since tertiary and higher order constraints will appear. We leave this problem for further research.

The paper is organized as follows. In Sec. II, we review the two kinds of mechanics, nonholonomic and vakonomic mechanics, from a unified variational approach. The constraint algorithm in its geometric version is described in Sec. III and applied to vakonomic mechanics in Secs. IV and V. In Sec. VI, we study the second-order differential problem and in Sec. VII, we classify the constraints according to Dirac. In Sec. VIII, we discuss what happens if the constraints are not globally defined on $T Q$.

\section{VARIATIONAL METHODS IN MECHANICS}

In this section we shall give a brief account of the variational principles involved in the derivation of the equations of motion in classical mechanics. For a more extended discussion see, for instance, Refs. 3, 8, 28 and 36.

Let $Q$ be an $n$-dimensional configuration manifold, and $L: T Q \rightarrow \mathrm{R}$ an autonomous Lagrangian function. If $\left(q^{A}\right)$ are coordinates on $Q$, we denote by $\left(q^{A}, \dot{q}^{A}\right)$ the natural bundle coordinates on $T Q$ such that the tangent bundle projection $\tau_{Q}: T Q \rightarrow Q$ reads as $\tau_{Q}\left(q^{A}, \dot{q}^{A}\right)=\left(q^{A}\right)$.

Given two points $x, y \in Q$ we define the manifold of twice differentiable curves joining $x$ and $y$ as

$$
\mathcal{C}^{2}(x, y)=\left\{c:[0,1] \rightarrow Q / c \text { is } C^{2}, c(0)=x \text { and } c(1)=y\right\} .
$$

Let $c$ be a curve in $\mathcal{C}^{2}(x, y)$. As is well known, the tangent space of $\mathcal{C}^{2}(x, y)$ at $c$ is given by

$$
T_{c} \mathcal{C}^{2}(x, y)=\left\{X:[0,1] \rightarrow T Q / X \text { is } C^{1}, X(t) \in T_{c(t)} Q, X(0)=0 \text { and } X(1)=0\right\} .
$$

We will assume here that $L$ is subjected to nonholonomic linear constraints given by a submanifold $M$ of $T Q$. Alternatively, the submanifold $M$ can be viewed as the total space of a vector subbundle of $T Q$, or, equivalently, as a distribution on $Q$ which will be denoted by the same letter.

Therefore, if the annihilator $M^{\circ}$ of $M$ is locally spanned by $m$ independent one-forms $\left\{\omega_{1}, \ldots, \omega_{m}\right\}$, where $\omega_{i}=\mu_{i A} d q^{A}$, we have that the constraint functions $\left\{\Phi_{1}, \ldots, \Phi_{m}\right\}$ are just the evaluation functions of this basis, that is, $\Phi_{i}\left(v_{q}\right)=\left\langle v_{q}, \omega_{i}(q)\right\rangle$ for all $v_{q} \in T_{q} Q, 1 \leqslant i \leqslant m$. Now, we introduce the submanifold of $\mathcal{C}^{2}(x, y)$ which consists of those curves which are compatible with the constraint submanifold $M$,

$$
\widetilde{\mathcal{C}}^{2}(x, y)=\left\{\widetilde{c} \in \mathcal{C}^{2}(x, y) / \dot{\tilde{c}}(t) \in M_{\tilde{c}(t)}, \forall t \in[0,1]\right\} .
$$

Given a curve $\widetilde{c} \in \widetilde{\mathcal{C}}^{2}(x, y)$, the constraints allow us to consider a special vector subspace of $T_{\bar{c}} \mathcal{C}^{2}(x, y)$,

$$
\mathcal{V}_{\tilde{c}}=\left\{X \in T_{\tilde{c}} \mathcal{C}^{2}(x, y) / \omega_{i}(X)=0,1 \leqslant i \leqslant m\right\}
$$


which are the allowed variations. Then, if $X=X^{A}\left(\partial / \partial q^{A}\right)$, we deduce that $X \in \mathcal{V}_{\tilde{c}}$ if and only if

$$
\mu_{i A} X^{A}=0, \quad \forall 1 \leqslant i \leqslant m,
$$

along the curve $\widetilde{c}$.

Next, define a functional $\mathcal{J}$ by

$$
\begin{gathered}
\mathcal{J}: \mathcal{C}^{2}(x, y) \rightarrow \mathrm{R} \\
c \mapsto \int_{0}^{1} L(\dot{c}(t)) d t .
\end{gathered}
$$

A direct computation using integration by parts shows that (see Ref. 8)

$$
d \mathcal{J}(c)(X)=\int_{0}^{1}\left(\frac{\partial L}{\partial q^{A}}-\frac{d}{d t}\left(\frac{\partial L}{\partial \dot{q}^{A}}\right)\right) X^{A} d t
$$

for $c \in \mathcal{C}^{2}(x, y)$ and $X \in T_{c} \mathcal{C}^{2}(x, y)$.

\section{A. Unconstrained systems}

In this case, $M=T Q$. The Hamilton principle states that a curve $c \in \mathcal{C}^{2}(x, y)$ is a motion of the Lagrangian system defined by $L$ if and only if $c$ is a critical point of $\mathcal{J}$; that is, iff $d \mathcal{J}(c)(X)$ $=0$ for all $X \in T_{c} \mathcal{C}^{2}(x, y)$, or

$$
\int_{0}^{1}\left(\frac{\partial L}{\partial q^{A}}-\frac{d}{d t}\left(\frac{\partial L}{\partial \dot{q}^{A}}\right)\right) X^{A} d t=0, \quad \forall X^{A} .
$$

This condition is equivalent to the Euler-Lagrange equations

$$
\frac{d}{d t}\left(\frac{\partial L}{\partial \dot{q}^{A}}\right)-\frac{\partial L}{\partial q^{A}}=0, \quad 1 \leqslant A \leqslant n .
$$

\section{B. Nonholonomic mechanics}

In this case, a curve $\widetilde{c} \in \widetilde{C}^{2}(x, y)$ is a motion if and only if it satisfies $d \mathcal{J}(\widetilde{c})(X)=0$, for all $X \in \mathcal{V}_{\tilde{c}}$, that is,

$$
\int_{0}^{1}\left(\frac{\partial L}{\partial q^{A}}-\frac{d}{d t}\left(\frac{\partial L}{\partial \dot{q}^{A}}\right)\right) X^{A} d t=0
$$

for all $X^{A}$ satisfying Eq. (1).

As before, we deduce that $\widetilde{c}$ is a motion if and only if

$$
\left(\frac{\partial L}{\partial q^{A}}-\frac{d}{d t}\left(\frac{\partial L}{\partial \dot{q}^{A}}\right)\right) X^{A}=0,
$$

for all $X^{A}$ satisfying Eq. (1), which is just the statement of d'Alembert's principle. Therefore, $\widetilde{c}$ is a motion for the nonholonomic system if and only if

$$
\frac{d}{d t}\left(\frac{\partial L}{\partial \dot{q}^{A}}\right)-\frac{\partial L}{\partial q^{A}}=-\lambda^{i} \mu_{i A}, \quad 1 \leqslant A \leqslant n,
$$

for some Lagrange multipliers $\lambda^{1}, \ldots, \lambda^{m}$. 


\section{Vakonomic mechanics}

In vakonomic mechanics, a curve $\widetilde{c} \in \widetilde{\mathcal{C}}^{2}(x, y)$ is a motion if and only if $d \mathcal{J}(\widetilde{c})(\widetilde{X})=0$, for all $\widetilde{X} \in T_{\widetilde{c}} \widetilde{C}^{2}(x, y)$, i.e., the motions are the extremals of the restriction of the functional to the curves satisfying the constraints.

Now, using the Lagrange multipliers theorem in an infinite dimensional context, we deduce (see Refs. 1, 3, 8, and 36) that $\widetilde{c}$ is an admissible regular motion if and only if there exist $m$ functions $\lambda^{1}, \ldots, \lambda^{m}, \lambda^{i}:[0,1] \rightarrow \mathbb{R}$ such that

$$
\frac{d}{d t}\left(\frac{\partial L}{\partial \dot{q}^{A}}\right)-\frac{\partial L}{\partial q^{A}}=-\lambda^{i}\left(\frac{\partial \mu_{i A}}{\partial q^{B}} \dot{q}^{B}-\frac{\partial \mu_{i B}}{\partial q^{A}} \dot{q}^{B}\right)-\frac{d \lambda^{i}}{d t} \mu_{i A}, \quad 1 \leqslant A \leqslant n
$$

An alternative approach to vakonomic mechanics is the following. From (4) we deduce that a curve $\widetilde{c}=\left(q^{A}(t)\right)$ in $\widetilde{\mathcal{C}}^{2}(x, y)$ is a solution of the vakonomic equations if and only if there exist local functions $\lambda^{1}, \ldots, \lambda^{m}$ on $\mathbb{R}$ such that $\bar{c}(t)=\left(q^{A}(t), \lambda^{i}(t)\right)$ is an extremal for the extended Lagrangian

$$
\mathcal{L}: T\left(Q \times \mathbb{R}^{m}\right) \rightarrow \mathbb{R}, \quad \mathcal{L}=L+\lambda^{i} \Phi_{i},
$$

i.e., it satisfies the Euler-Lagrange equations

$$
\begin{aligned}
& \frac{d}{d t}\left(\frac{\partial \mathcal{L}}{\partial \dot{q}^{A}}\right)-\frac{\partial \mathcal{L}}{\partial q^{A}}=0, \quad 1 \leqslant A \leqslant n, \\
& \frac{d}{d t}\left(\frac{\partial \mathcal{L}}{\partial \dot{\lambda}^{i}}\right)-\frac{\partial \mathcal{L}}{\partial \lambda^{i}}=\Phi_{i}\left(q^{A}, \dot{q}^{A}\right)=0, \quad 1 \leqslant i \leqslant m
\end{aligned}
$$

(see Refs. 1, 3, 8, and 36 for details).

\section{THE CONSTRAINT ALGORITHM}

First of all, let us recall the geometric formulation for Lagrangian mechanics (see Ref. 37). Let $S=\partial / \partial \dot{q}^{A} \otimes d q^{A}$ be the canonical almost tangent structure on $T Q$ and $\Delta=\dot{q}^{A}\left(\partial / \partial \dot{q}^{A}\right)$ the Liouville vector field on $T Q$. From the Lagrangian $L$, we construct the Poincaré-Cartan two-form $\omega_{L}=-d S^{*}(d L)$ and the energy $E_{L}=\Delta(L)-L$.

Then, the equations of motion can be equivalently written as

$$
i_{x} \omega_{L}=d E_{L}
$$

Indeed, if the Lagrangian $L$ is regular, i.e., its Hessian matrix $\operatorname{Hess}(L)=\left(\partial^{2} L / \partial \dot{q}^{A} \partial \dot{q}^{B}\right)$ is not singular, then $\omega_{L}$ is symplectic, and (5) has a unique solution $\Gamma_{L}$ which is a second-order differential equation (SODE). The solutions of $\Gamma_{L}$ are just the ones of the Euler-Lagrange equations. If $L$ is not regular, then (5) has no solution in general, and even if a solution exists, it will not be unique or a SODE.

In order to treat with this kind of system, Gotay and Nester ${ }^{31-33}$ developed a constraint algorithm (a geometrization of the Dirac-Bergmann algorithm), applicable in the general framework of presymplectic manifolds as is described in the following. A presymplectic system is a triple, $(\mathcal{M}, \omega, \alpha)$, that consists of a smooth manifold $\mathcal{M}$, a closed two-form $\omega$ with constant rank, and a closed one-form $\alpha$.

We are interested in searching the possible solutions of

$$
i_{x} \omega=\alpha .
$$

Let $b: T \mathcal{M} \rightarrow T^{*} \mathcal{M}$ be the map defined by $b(X)=i_{X} \omega$. If $\omega$ is not symplectic, then $b$ is not surjective and, consequently, (6) has no global solution on $\mathcal{M}$ in general. 
Consider the points of $\mathcal{M}$ where (6) has a solution and assume that this set is a submanifold $\mathcal{M}_{2}$ of $\mathcal{M}_{1}=\mathcal{M}$ (this will be our case, since we are assuming that $\omega$ has constant rank). It could still happen that the solutions on $\mathcal{M}_{2}$ are not tangent to $\mathcal{M}_{2}$. In consequence, we take a submanifold $\mathcal{M}_{3}$ of $\mathcal{M}_{2}$ where the solutions are tangent to $\mathcal{M}_{2}$. Continuing with this process repeatedly, we generate a sequence of submanifolds

$$
\cdots \hookrightarrow \mathcal{M}_{i} \cdots \hookrightarrow \mathcal{M}_{2} \cdots \hookrightarrow \mathcal{M}_{1}=\mathcal{M},
$$

in such a way that if the algorithm stabilizes for some $k$, i.e., $\mathcal{M}_{k}=\mathcal{M}_{k+1} \equiv \mathcal{M}_{f}$, then there exists a vector field $\Gamma$ on $\mathcal{M}_{f}$ such that

$$
\left(i_{\Gamma} \omega=\alpha\right)_{/ \mathcal{M}_{f}}
$$

Notice that if we finish the process at the step $k=1$, it will mean that there is a global solution $\Gamma$ on the whole of $\mathcal{M}$.

Alternatively, the above submanifolds can be obtained as follows:

$$
\mathcal{M}_{i}=\left\{x \in \mathcal{M} / \alpha(x)(z)=0, \forall z \in T_{x} \mathcal{M}_{i-1}^{\perp}\right\},
$$

where

$$
T_{x} \mathcal{M}_{i-1}^{\perp}=\left\{z \in T_{x} \mathcal{M} / \omega(x)(v, z)=0, \forall v \in T_{x} \mathcal{M}_{i-1}\right\} .
$$

We call $\mathcal{M}_{2}$ the secondary constraint submanifold, $\mathcal{M}_{3}$ the tertiary constraint submanifold, and in general $\mathcal{M}_{i}$ will be the $i$-ary constraint submanifold. If the algorithm stabilizes, then $\mathcal{M}_{f}$ will be the final constraint submanifold. Accordingly, the (local) functions defining these submanifolds will be termed secondary constraints, ternary constraints, and so on.

\section{THE LAGRANGIAN FORMALISM}

Let $Q$ be an $n$-dimensional manifold representing the configuration space of a mechanical system described by a Lagrangian function $L: T Q \rightarrow \mathbb{R}$ and subjected to linear nonholonomic constraints given by a submanifold $M$ of $T Q$.

We shall assume that the Lagrangian is of natural type, that is $L=T-U$, where $T$ is the kinetic energy of a Riemannian metric $g$ on $Q$, and $U: Q \rightarrow \mathbb{R}$ is a potential energy.

In bundle coordinates $L$ reads as

$$
L\left(q^{A}, \dot{q}^{A}\right)=\frac{1}{2} g_{A B}(q) \dot{q}^{A} \dot{q}^{B}-U(q) .
$$

As we have seen earlier, the constraint submanifold $M$ is locally defined as the zero set of $m$ independent linear nonholonomic constraints $\Phi_{i}\left(q^{A}, \dot{q}^{A}\right)=\mu_{i A}(q) \dot{q}^{A}$.

For the sake of simplicity, we shall assume that the constraints $\Phi_{i}$ are globally defined on the whole $T Q$. Later, we shall consider the general case.

Consider the product manifold $P=Q \times \mathbb{R}^{m}$ with local coordinates $\left(q^{A}, \lambda^{i}\right)$. As we have seen in Sec. II, the equations of motion corresponding to the vakonomic problem given by $L$ and $M$ can be formulated in terms of the extended lagrangian $\mathcal{L}: T P \rightarrow \mathbb{R}, \mathcal{L}=L+\lambda^{i} \Phi_{i}$.

In what follows, we will identify $T P$ with $T Q \times T R^{m}$, and denote by $\pi_{1}: T Q \times T R^{m} \rightarrow T Q$ and $\pi_{2}: T Q \times T \mathbb{R}^{m} \rightarrow T \mathbb{R}^{m}$ the canonical projections of $T Q \times T \mathbb{R}^{m}$ onto $T Q$ and $T \mathbb{R}^{m}$, respectively.

The Poincaré-Cartan two-form $\omega_{\mathcal{L}}$ associated to $\mathcal{L}$ is

$$
\omega_{\mathcal{L}}=\left(\frac{\partial g_{A C}}{\partial q^{B}} \dot{q}^{C}+\lambda^{i} \frac{\partial \mu_{i A}}{\partial q^{B}}\right) d q^{A} \wedge d q^{B}+\mu_{i A} d q^{A} \wedge d \lambda^{i}+g_{B A} d q^{A} \wedge d \dot{q}^{B}
$$


Notice that $\omega_{\mathcal{L}}$ is not symplectic because of the singular character of $\mathcal{L}$. Indeed, $\partial \mathcal{L} / \partial \lambda^{i}=0$. However, it still has constant rank as shows its Hessian matrix

$$
\operatorname{Hess}(\mathcal{L})=\left(\begin{array}{cc}
\frac{\partial^{2} \mathcal{L}}{\partial \dot{q}^{A} \partial \dot{q}^{B}} & \frac{\partial^{2} \mathcal{L}}{\partial \dot{\lambda}^{i} \partial \dot{q}^{B}} \\
\frac{\partial^{2} \mathcal{L}}{\partial \dot{q}^{A} \partial \dot{\lambda}^{j}} & \frac{\partial^{2} \mathcal{L}}{\partial \dot{\lambda}^{i} \partial \dot{\lambda}^{j}}
\end{array}\right)=\left(\begin{array}{cc}
\operatorname{Hess}(L) & 0 \\
0 & 0
\end{array}\right)
$$

Therefore, we have

$$
\operatorname{rank}\left(\omega_{\mathcal{L}}\right)=\operatorname{rank}(\operatorname{Hess}(\mathcal{L}))=\operatorname{rank}(\operatorname{Hess}(L))=\operatorname{rank}\left(\omega_{L}\right)=2 n .
$$

We deduce that the triple $\left(T P, \omega_{\mathcal{L}}, d E_{\mathcal{L}}\right)$ is a presymplectic system, with $E_{\mathcal{L}}=\Delta(\mathcal{L})-\mathcal{L}$ the energy of $\mathcal{L}$.

In this presymplectic framework the equations of motion are written as

$$
i_{X} \omega_{\mathcal{L}}=d E_{\mathcal{L}} .
$$

Next, we will apply Gotay and Nester's algorithm described in Sec. III to find a solution of (7).

Put $P_{1}=T P$, then

$$
P_{2}=\left\{x \in P_{1} /\left\langle d E_{\mathcal{L}}, Z\right\rangle(x)=0, \forall Z \in\left(T_{x} P_{1}\right)^{\perp}\right\},
$$

where

$$
\left(T_{x} P_{1}\right)^{\perp}=\left\{Z \in T_{x} P_{1} / \omega_{\mathcal{L}}(Z, W)=0, \forall W \in T_{x} P_{1}\right\}=\left\{Z \in T_{x} P_{1} / b_{\mathcal{L}}(Z)=0\right\} .
$$

Thus, to obtain $P_{2}$ we need first to calculate ker $b_{\mathcal{L}}$.

A direct computation shows that

$$
\frac{{ }^{i} \partial}{\partial \lambda^{i}} \omega_{\mathcal{L}}=0 .
$$

Moreover, we also have

$$
{ }^{i} Z_{i} \omega_{\mathcal{L}}=0,
$$

where

$$
Z_{i}=\frac{\partial}{\partial \lambda^{i}}-g^{B C} \mu_{i C} \frac{\partial}{\partial \dot{q}^{B}}, \quad 1 \leqslant i \leqslant m
$$

Therefore, since the vector fields $\left\{\partial / \partial \lambda^{i}, Z_{i}\right\}$ are linearly independent and rank $\omega_{\mathcal{L}}=2 n$, we deduce that they generate $\operatorname{ker} b_{\mathcal{L}}$, that is,

$$
\operatorname{ker} b_{\mathcal{L}}=\operatorname{span}\left\{Z_{i}, \frac{\partial}{\partial \lambda^{-i}}\right\} .
$$

Remark IV.1: It is not difficult to see that

$$
\operatorname{dim}\left(\operatorname{ker} b_{\mathcal{L}}\right)=2 \operatorname{dim}\left(V(T P) \cap \operatorname{ker} b_{\mathcal{L}}\right),
$$


where $V(T P)$ is the vertical bundle over $P$. Therefore, $\mathcal{L}$ is a singular Lagrangian of Type II according to the classification in Refs. 38 and 39.

Notice that $E_{\mathcal{L}}=\left(\pi_{1}\right) *\left(E_{L}\right)$, where $E_{L}$ is the energy corresponding to the Lagrangian $L$. In what follows, we will write $E_{L}$ instead of $\left(\pi_{1}\right)^{*}\left(E_{L}\right)$, for brevity.

Now, in order to compute the constraint functions which define $P_{2}$, we calculate $\left(d E_{\mathcal{L}}\right)_{x}\left(\partial / \partial \lambda^{i}\right)$ and $\left(d E_{\mathcal{L}}\right)_{x}\left(Z_{i}\right), 1 \leqslant i \leqslant m$

$$
\begin{gathered}
\left(d E_{\mathcal{L}}\right)\left(\frac{\partial}{\partial \dot{\lambda}^{i}}\right)=\frac{\partial E_{L}}{\partial \dot{\lambda}^{i}}=0, \\
\left(d E_{\mathcal{L}}\right)\left(Z_{i}\right)=Z_{i}\left(E_{\mathcal{L}}\right)=\left(\frac{\partial}{\partial \lambda^{i}}-g^{B C} \mu_{i C} \frac{\partial}{\partial \dot{q}^{B}}\right)\left(\frac{\partial L}{\partial \dot{q}^{A}} \dot{q}^{A}-L\right) \\
=-g^{B C} \mu_{i C} \frac{\partial L}{\partial \dot{q}^{B}}+g^{B C} \mu_{i C} \frac{\partial L}{\partial \dot{q}^{B}}-g^{B C} \mu_{i C} g_{A B} \dot{q}^{A}=-\mu_{i A} \dot{q}^{A},
\end{gathered}
$$

which are the original constraints.

Thus, we have

$$
P_{2}=\left\{x \in P_{1} / \Phi_{i}\left(\pi_{1}(x)\right)=0,1 \leqslant i \leqslant m\right\} .
$$

Next, we shall compute $T P_{2}$. Take $X$ a vector field tangent to $P_{2}$, that is, if

$$
X=X_{1}^{A} \frac{\partial}{\partial q^{A}}+X_{2}^{i} \frac{\partial}{\partial \lambda^{i}}+X_{3}^{A} \frac{\partial}{\partial \dot{q}^{A}}+X_{4}^{i} \frac{\partial}{\partial \lambda^{i}},
$$

we have

$$
X\left(\Phi_{i}\right)=X_{1}^{A} \dot{q}^{B} \frac{\partial \mu_{i B}}{\partial q^{A}}+X_{3}^{A} \mu_{i A}=0, \quad \forall i
$$

The matrix $\left(\mu_{i A}\right)$ has rank $m$, so we can assume that the submatrix $\left(\mu_{i j}\right), 1 \leqslant i, j \leqslant m$ is invertible, with inverse matrix $\left(\mu^{j i}\right)$. Equation (8) can be written as

$$
X_{3}^{j} \mu_{i j}+X_{3}^{a} \mu_{i a}=-X_{1}^{A} \dot{q}^{B} \frac{\partial \mu_{i B}}{\partial q^{A}},
$$

where $1 \leqslant i, j \leqslant m$ and $m+1 \leqslant a \leqslant n$. Now, multiplying by $\left(\mu^{j i}\right)$ we obtain that

$$
X_{3}^{j}=-\mu^{j i} X_{1}^{A} \dot{q}^{B} \frac{\partial \mu_{i B}}{\partial q^{A}}-\mu^{j i} X_{3}^{a} \mu_{i a}
$$

Consequently, we deduce that $T P_{2}$ is spanned by the vector fields

$$
\left\{\frac{\partial}{\partial \lambda^{i}}, \frac{\partial}{\partial \dot{\lambda}^{i}}, \frac{\partial}{\partial q^{A}}-\dot{q}^{B} \frac{\partial \mu_{i B}}{\partial q^{A}} \mu^{j i} \frac{\partial}{\partial \dot{q}^{j}}, \frac{\partial}{\partial \dot{q}^{a}}-\mu^{j i} \mu_{i a} \frac{\partial}{\partial \dot{q}^{j}}\right\} .
$$

Next, we want to compute $T P_{2}^{\perp}$. Consider a vector field $Y$,

$$
Y=Y_{1}^{A} \frac{\partial}{\partial q^{A}}+Y_{2}^{i} \frac{\partial}{\partial \lambda^{i}}+Y_{3}^{A} \frac{\partial}{\partial \dot{q}^{A}}+Y_{4}^{i} \frac{\partial}{\partial \dot{\lambda}^{i}},
$$


such that $Y \in T P_{2}^{\perp}$. After some calculations, we obtain that

$$
\begin{gathered}
Y_{1}^{A}=0, \\
Y_{3}^{A}=-g^{E A} \mu_{i E} Y_{2}^{i} .
\end{gathered}
$$

Then

$$
d E_{L}(Y)=g_{A B} \dot{q}^{B} Y_{3}^{A}=-g_{A B} \dot{q}^{B} g^{E A} \mu_{i E} Y_{2}^{i}=-\dot{q}^{E} \mu_{i E} Y_{2}^{i}=\Phi_{i} Y_{2}^{i}=0,
$$

on $P_{2}$ and, therefore, $P_{3}=P_{2}$. This means that the algorithm stabilizes at $P_{2}$, and $P_{2}$ is the final constraint submanifold.

Our aim in the rest of this section is to get explicit expressions for the solutions of Eq. (7). For that purpose, take an arbitrary vector field $\Gamma$ on $T P$ locally written as

$$
\Gamma=\mathcal{A}^{A} \frac{\partial}{\partial q^{A}}+\mathcal{B}^{i} \frac{\partial}{\partial \lambda^{i}}+\mathcal{C}^{A} \frac{\partial}{\partial \dot{q}^{A}}+\mathcal{D}^{i} \frac{\partial}{\partial \dot{\lambda}^{i}},
$$

and assume that it satisfies

$$
i_{\Gamma} \omega_{\mathcal{L}}=d E_{\mathcal{L}} .
$$

A straightforward computation shows that

$$
\begin{gathered}
i_{\Gamma} \omega_{\mathcal{L}}=\left[\mathcal{A}^{B}\left[\left(\frac{\partial g_{B C}}{\partial q^{A}}-\frac{\partial g_{A C}}{\partial q^{B}}\right) \dot{q}^{C}+\lambda^{i}\left(\frac{\partial \mu_{i B}}{\partial q^{A}}-\frac{\partial \mu_{i A}}{\partial q^{B}}\right)\right]-\mathcal{B}^{i} \mu_{i A}-\mathcal{C}^{B} g_{A B}\right] d q^{A} \\
+\mathcal{A}^{A} \mu_{i A} d \lambda^{i}+\mathcal{A}^{A} g_{A B} d \dot{q}^{B} \\
d E_{\mathcal{L}}=\left[\frac{1}{2} \frac{\partial g_{B C}}{\partial q^{A}} \dot{q}^{C} \dot{q}^{B}+\frac{\partial U}{\partial q^{A}}\right] d q^{A}+g_{A B} \dot{q}^{B} d \dot{q}^{A} .
\end{gathered}
$$

Comparing the coefficients of $d \dot{q}^{B}$ and $d \lambda^{i}$ we deduce that

$$
\mathcal{A}^{B} g_{A B}=\dot{q}^{B} g_{B A}, \quad \mathcal{A}^{A} \mu_{i A}=0,
$$

which implies $\mathcal{A}^{A}=\dot{q}^{A}, 1 \leqslant A \leqslant n$, and

$$
\mu_{i A} \dot{q}^{A}=0, \quad 1 \leqslant i \leqslant m .
$$

Comparing now the coefficients of $d q^{A}$, we find that $\mathcal{B}^{i}$ and $\mathcal{C}^{B}$ are related as follows:

$$
\mathcal{B}^{i} \mu_{i A}+\mathcal{C}^{B} g_{A B}=\lambda^{i} \dot{q}^{D}\left(\frac{\partial \mu_{i D}}{\partial q^{A}}-\frac{\partial \mu_{i A}}{\partial q^{D}}\right)+\left(\frac{1}{2} \frac{\partial g_{D C}}{\partial q^{A}}-\frac{\partial g_{A C}}{\partial q^{D}}\right) \dot{q}^{C} \dot{q}^{D}-\frac{\partial U}{\partial q^{A}},
$$

or, equivalently,

$$
\mathcal{C}^{B}=g^{A B} \dot{q}^{D}\left[\lambda^{i}\left(\frac{\partial \mu_{i D}}{\partial q^{A}}-\frac{\partial \mu_{i A}}{\partial q^{D}}\right)+\left(\frac{1}{2} \frac{\partial g_{C D}}{\partial q^{A}}-\frac{\partial g_{A C}}{\partial q^{D}}\right) \dot{q}^{C}\right]-g^{A B} \frac{\partial U}{\partial q^{A}}-g^{A B} \mu_{i A} \mathcal{B}^{i} .
$$

Moreover, since $\Gamma$ has to be tangent to $P_{2}$, we get

$$
\mathcal{C}^{B} \mu_{j B}+\dot{q}^{A} \dot{q}^{B} \frac{\partial \mu_{j B}}{\partial q^{A}}=0 .
$$

Introducing the expression for $\mathcal{C}^{B}$ obtained in (11) into (12), we have 


$$
\begin{aligned}
g^{A B} \mu_{j B} \mu_{i A} \mathcal{B}^{i}= & \dot{q}^{A} \dot{q}^{B} \frac{\partial \mu_{j B}}{\partial q^{A}}+\mu_{j B} g^{A B} \dot{q}^{D}\left[\lambda^{i}\left(\frac{\partial \mu_{i D}}{\partial q^{A}}-\frac{\partial \mu_{i A}}{\partial q^{D}}\right)\right. \\
& \left.+\left(\frac{1}{2} \frac{\partial g_{C D}}{\partial q^{A}}-\frac{\partial g_{A C}}{\partial q^{D}}\right) \dot{q}^{C}\right]-\mu_{j B} g^{A B} \frac{\partial U}{\partial q^{A}}
\end{aligned}
$$

But the matrix $D=\left(D_{i j}\right)$, with

$$
D_{i j}=g^{A B} \mu_{i A} \mu_{j B},
$$

is regular (see Refs. 16 and 17), so $\mathcal{B}^{i}$ is explicitly given as

$$
\begin{aligned}
\mathcal{B}^{i}= & D^{i j} \dot{q}^{A} \dot{q}^{B} \frac{\partial \mu_{j B}}{\partial q^{A}}+D^{i j} \mu_{j B} g^{A B} \dot{q}^{D}\left[\lambda^{k}\left(\frac{\partial \mu_{k D}}{\partial q^{A}}-\frac{\partial \mu_{k A}}{\partial q^{D}}\right)\right. \\
& \left.+\left(\frac{1}{2} \frac{\partial g_{D C}}{\partial q^{A}}-\frac{\partial g_{A C}}{\partial q^{D}}\right) \dot{q}^{C}\right]-D^{i j} \mu_{j B} g^{A B} \frac{\partial U}{\partial q^{A}},
\end{aligned}
$$

where $\left(D^{i j}\right)$ is the inverse matrix of $D$.

Therefore, from (11) we obtain an explicit formula for $\mathcal{C}^{B}$,

$$
\begin{aligned}
\mathcal{C}^{B}= & g^{A B} \dot{q}^{D}\left[\lambda^{k}\left(\frac{\partial \mu_{k D}}{\partial q^{A}}-\frac{\partial \mu_{k A}}{\partial q^{D}}\right)+\left(\frac{1}{2} \frac{\partial g_{C D}}{\partial q^{A}}-\frac{\partial g_{A C}}{\partial q^{D}}\right) \dot{q}^{C}\right]-g^{A B} \frac{\partial U}{\partial q^{A}}-g^{A B} \mu_{i A}\left[D^{i j} \dot{q}^{E} \dot{q}^{F} \frac{\partial \mu_{j F}}{\partial q^{E}}\right. \\
& \left.+D^{i j} \mu_{j F} g^{E F} \dot{q}^{D}\left[\lambda^{k}\left(\frac{\partial \mu_{k D}}{\partial q^{E}}-\frac{\partial \mu_{k E}}{\partial q^{D}}\right)+\left(\frac{1}{2} \frac{\partial g_{D C}}{\partial q^{E}}-\frac{\partial g_{E C}}{\partial q^{D}}\right) \dot{q}^{C}\right]-D^{i j} \mu_{j F} g^{E F} \frac{\partial U}{\partial q^{E}}\right]
\end{aligned}
$$

Summing up, a vector field $\Gamma$ with local expression

$$
\Gamma=\dot{q}^{A} \frac{\partial}{\partial q^{A}}+\mathcal{B}^{i} \frac{\partial}{\partial \lambda^{i}}+\mathcal{C}^{A} \frac{\partial}{\partial \dot{q}^{A}}+\mathcal{D}^{i} \frac{\partial}{\partial \lambda^{i}},
$$

satisfies the conditions

$$
\begin{gathered}
\left(i_{\Gamma} \omega_{\mathcal{L}}=d E_{\mathcal{L}}\right)_{\mid P_{2}}, \\
\Gamma \in T P_{2},
\end{gathered}
$$

if and only if the coefficients $\mathcal{B}^{i}$ and $\mathcal{C}^{B}$ satisfy (14) and (15), respectively. The parameters $\mathcal{D}^{i}$ remain undetermined and give rise to a family $\Gamma_{\mathcal{D}}$ of vector fields satisfying the above-mentioned system.

Remark IV.2: The solutions we have obtained do not satisfy the SODE condition along $P_{2}$ since $S(\Gamma) \neq \Delta$, that is, $\mathcal{B}^{i} \neq \dot{\lambda}^{i}, 1 \leqslant i \leqslant m$. In the next sections, we will find a submanifold $\mathcal{S}$ of $P_{2}$ and a vector field $\widetilde{\Gamma}$ on it such that $\left(i_{\Gamma} \omega_{\mathcal{L}}=d E_{\mathcal{L}}\right)_{\mathcal{S}}$ and $(S(\widetilde{\Gamma})=\Delta)_{\mid \mathcal{S}}$ hold simultaneously. The existence of this submanifold can be ensured if a certain admissibility condition is fullfilled (see Refs. 31 and 33).

We are now in a position to make a first comparison between what we have obtained for $\left(T P, \omega_{\mathcal{L}}, d E_{\mathcal{L}}\right)$ by means of the presymplectic formalism and the vakonomic formulation for the original Lagrangian $L$.

First of all, the final constraint submanifold $P_{2}$ and $M$ are closely related. Indeed, $P_{2}$ and $M \times T \mathrm{R}^{m}$ are diffeomorphic in a natural way. Moreover, let $\Gamma$ be a vector field on $P_{2}$ such that $i_{\Gamma} \omega_{\mathcal{L}}=d E_{\mathcal{L}}$. Since $T P_{2}$ is diffeomorphic to $T M \times T T R^{m}$, then $\Gamma$ splits as $\Gamma=(X, Z)$, with $X$ $=X_{\lambda}: M \rightarrow T M$ and $Z=Z_{(q, \dot{q})}: T \mathbb{R}^{m} \rightarrow T T \mathbb{R}^{m}$ vector fields on $M$ and $T \mathbb{R}^{m}$ depending on the parameters $\lambda$ and $(q, \dot{q})$, respectively. 
The obstacle for the above-mentioned splitting to be "clean," that is, $X$ being independent of $\lambda$ and $Z$ being independent of $(q, \dot{q})$, is the coupling of the coordinates $(q, \dot{q})$ and $\lambda$ in the vakonomic equations, a fact that can also be seen in the explicit expressions for $\mathcal{B}^{i}=\mathcal{B}^{i}(q, \lambda, \dot{q})$ and $\mathcal{C}^{B}=\mathcal{C}^{B}(q, \lambda, \dot{q})$ [see (14) and (15)]. A look to these local expressions shows that if the crossed terms $\partial \mu_{i B} / \partial q^{A}-\partial \mu_{i A} / \partial q^{B}$ vanish, then we will be able to project "cleanly" $\Gamma$ onto a vector field $X$ independent of parameters. Of course, this is just the case when the constraints are holonomic. $^{8}$

On the other hand, this can also be done for some mechanical systems subjected to nonholonomic constraints: for example, whenever we can get an expression for the Lagrange multipliers $\left(\lambda^{i}(t)\right)$ along solutions $\left(q^{A}(t), \dot{q}^{A}(t)\right)$. This is the case of the vertical rolling disk (see Example VII.4). In fact, we have that $X_{\lambda_{0}(t)}=\Gamma_{L, M}$, where $\left(\lambda_{0}^{i}(t)\right)$ is a special curve of Lagrange multipliers and $\Gamma_{L, M}$ is the nonholonomic vector field along $M$. Consequently, the solutions of the nonholonomic problem may be regarded as a subset of the vakonomic ones. ${ }^{8,24}$ As a by-product of the application of the Gotay and Nester algorithm, we have found a geometric characterization of this fact. However, it will not be true in general as pointed out in Ref. 8 and the question of when this can be done is still unanswered.

\section{THE HAMILTONIAN FORMALISM}

In this section, we will discuss the vakonomic system within the framework of the cotangent bundle $T^{*} P$. First of all, note that the Lagrangian $\mathcal{L}$ is almost regular, so we are just in the assumptions of Gotay and Nester. ${ }^{31,32}$

Our interest in developing this formulation is to classify the constraints appeared in the process following Dirac's criterion and, then, to define a Dirac bracket giving the evolution of dynamical variables.

Consider the Legendre transformation of $\mathcal{L}$,

$$
\mathcal{F} \mathcal{L}: T P \rightarrow T^{*} P \text {. }
$$

As is well known, the Legendre mapping is a fibered mapping over $P$, i.e., $\pi_{P} \circ \mathcal{F} \mathcal{L}=\tau_{P}$, where $\pi_{P}: T^{*} P \rightarrow P$ is the canonical projection. In local coordinates the Legendre transformation reads as

$$
\mathcal{F} \mathcal{L}\left(q^{A}, \lambda^{i}, \dot{q}^{A}, \dot{\lambda}^{i}\right)=\left(q^{A}, \lambda^{i},\left(\frac{\partial L}{\partial \dot{q}^{A}}\right)_{\left(q^{\mathcal{A}}, \dot{q}^{\mathcal{A}}\right)}+\lambda^{i}\left(\frac{\partial \Phi_{i}}{\partial \dot{q}^{A}}\right)_{\left(q^{\mathcal{A}}, \dot{q}^{\mathcal{A}}\right)}, 0\right) .
$$

Therefore, if $\left(q^{A}, \lambda^{i}, \hat{p}_{A}, \hat{p}_{i}\right)$ are bundle coordinates in $T^{*} P$ we have

$$
\hat{p}_{A}=g_{A B} \dot{q}^{B}+\lambda^{i} \mu_{i A}, \quad \hat{p}_{i}=0,
$$

along the image of $\mathcal{F} \mathcal{L}$.

Next we will prove that $\mathcal{L}$ is almost regular according to the definition in Refs. 31 and 32.

Proposition V.1: The following statements are true

(i) $\mathcal{F} \mathcal{L}(T P)=M_{1}$ is a submanifold of $T^{*} P$.

(ii) $F \mathcal{L}$ is a submersion on its image and its fibers are connected submanifolds of TP. Therefore, $\mathcal{L}$ is almost regular.

Proof: The Jacobian matrix of $\mathcal{F} \mathcal{L}$ is

$$
\left(\begin{array}{cccc}
I_{n} & 0 & K & 0 \\
0 & I_{m} & \bar{K} & 0 \\
0 & 0 & \operatorname{Hess}(L) & 0 \\
0 & 0 & 0 & 0
\end{array}\right),
$$


where $K=\left[\dot{q}^{C}\left(\partial g_{B C} / \partial q^{A}\right)+\lambda^{i}\left(\partial \mu_{i B} / \partial q^{A}\right)\right]$ and $\bar{K}=\left(\mu_{i A}\right)$. Then, rank $\mathcal{F} \mathcal{L}=2 n+m$ at every $x$ $\in T P$, and from the rank theorem we deduce that $M_{1}$ is a submanifold of $T^{*} P$. Moreover, with this differentiable structure the mapping $\mathcal{F} \mathcal{L}: T P \rightarrow M_{1}$ is a submersion.

Next, we will prove that $\mathcal{F} \mathcal{L}^{-1}(y)=\operatorname{span}\left\{\left(\partial / \partial \lambda^{i}\right)_{\pi_{P}(y)}\right\}$, for all $y \in M_{1}$. In this case, the fibers of $\mathcal{F} \mathcal{L}$ would be connected. Indeed, let $x_{1}, x_{2} \in \mathcal{F} \mathcal{L}^{-1}(y)$. Then both are in the same fiber of $T P$, i.e., $\tau_{P}\left(x_{1}\right)=\tau_{P}\left(x_{2}\right)$, and from the definition of $\mathcal{F} \mathcal{L}$ we deduce that $\mathcal{F} L\left(\pi_{1}\left(x_{1}\right)\right)=\mathcal{F} L\left(\pi_{1}\left(x_{2}\right)\right)$. Therefore $\pi_{1}\left(x_{1}\right)=\pi_{1}\left(x_{2}\right)$ since $\mathcal{F} L$ is a diffeomorphism. Consequently, $x_{1}$ and $x_{2}$ differ only in their components $\dot{\lambda}^{i}$. Thus, we have completed the proof.

Notice that $M_{1}$ is locally defined by the equations $\hat{p}_{i}=0$ for all $i$. Denote by $\omega_{1}=j_{1}^{*} \omega_{P}$, where $\omega_{P}=d q^{A} \wedge d \hat{p}_{A}+d \lambda^{i} \wedge d \hat{p}_{i}$ is the canonical symplectic form on $T^{*} P$ and $j_{1}: M_{1} \rightarrow T^{*} P$ is the canonical inclusion. Then

$$
\omega_{1}=d q^{A} \wedge d \hat{p}_{A}
$$

is a closed two-form on $M_{1}$ with constant rank $2 n<\operatorname{dim} M_{1}$.

Since $\mathcal{L}$ is almost regular, the energy $E_{\mathcal{L}}$ is constant along the fibers of $\mathcal{F} \mathcal{L}$ and it induces a well-defined function $h_{1}: M_{1} \rightarrow \mathbb{R}$ by the relation $h_{1} \circ \mathcal{F} \mathcal{L}=E_{\mathcal{L}}$. In fact,

$$
h_{1}\left(q^{A}, \lambda^{i}, \hat{p}_{A}, 0\right)=\frac{1}{2} g^{A B}\left(\hat{p}_{A}-\lambda^{i} \mu_{i A}\right)\left(\hat{p}_{B}-\lambda^{j} \mu_{j B}\right)+U(q) .
$$

Thus, the system $\left(M_{1}, \omega_{1}, d h_{1}\right)$ is presymplectic and we can apply to it the constraint algorithm. It should be noticed that Gotay and Nester's equivalence theorem (see Refs. 31 and 32) implies that this algorithm will stabilize at a submanifold $M_{2}$ of $M_{1}$ so that the following diagram

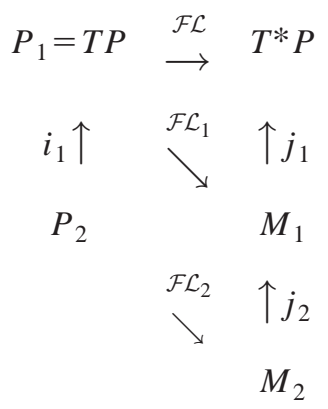

is conmutative. Here, $i_{1}$ and $j_{2}$ are the canonical inclusions, and $\mathcal{F} \mathcal{L}_{k}=\mathcal{F} \mathcal{L}_{\mid P_{k}}$ are submersions on their images $M_{k}$ for $k=1,2$.

The primary constraints are those defining $M_{1}$, that is, $\hat{p}_{i}=0$. In order to calculate the secondary constraints which in turn define $M_{2}$, we first compute

$$
\operatorname{ker}\left(\omega_{1}\right)_{y}=\left(T_{y} M_{1}\right)^{\perp}=\left\{\zeta \in T_{y} M_{1} /\left(\omega_{1}\right)_{y}(\zeta, \eta)=0, \forall \eta \in T_{y} M_{1}\right\} .
$$

In terms of the induced coordinate system on $M_{1}$, the tangent space of $M_{1}$ at $y$ is locally generated by

$$
\left\{\left(\frac{\partial}{\partial q^{A}}\right)_{y},\left(\frac{\partial}{\partial \lambda^{i}}\right)_{y},\left(\frac{\partial}{\partial \hat{p}_{A}}\right)\right\}_{y}
$$

If

$$
\zeta=\zeta_{1}^{A}\left(\frac{\partial}{\partial q^{A}}\right)_{y}+\zeta_{2}^{i}\left(\frac{\partial}{\partial \lambda^{i}}\right)_{y}+\zeta_{3}^{A}\left(\frac{\partial}{\partial \hat{p}_{A}}\right)_{y} \in T_{y} M_{1}
$$




$$
\eta=\eta_{1}^{A}\left(\frac{\partial}{\partial q^{A}}\right)_{y}+\eta_{2}^{i}\left(\frac{\partial}{\partial \lambda^{i}}\right)_{y}+\eta_{3}^{A}\left(\frac{\partial}{\partial \hat{p}_{A}}\right)_{y} \in T_{y} M_{1}
$$

then we have

$$
\left(\omega_{1}\right)_{y}(\zeta, \eta)=\left(d q^{A} \wedge d \hat{p}_{A}\right)_{y}(\zeta, \eta)=\zeta_{1}^{A} \eta_{3}^{A}-\zeta_{3}^{A} \eta_{1}^{A}=0, \quad \forall \eta_{1}^{A}, \eta_{3}^{A}
$$

Thus $\zeta_{1}^{A}=\zeta_{3}^{A}=0$, which implies that

$$
\left(T_{y} M_{1}\right)^{\perp}=\operatorname{span}\left\{\left(\frac{\partial}{\partial \lambda_{i}}\right)_{y}\right\}
$$

Then $d h_{1}\left(\partial / \partial \lambda^{i}\right)=\left(\partial h_{1} / \partial \lambda^{i}\right)=-\chi_{i}$ provides the new constraints

$$
\chi_{i}=\mu_{i A} g^{A B}\left(\hat{p}_{B}-\lambda^{j} \mu_{j} B\right), \quad 1 \leqslant i \leqslant m .
$$

Consequently, $M_{2}$ is defined by the constraints $\hat{p}_{i}(y)=0$ and $\chi_{i}(y)=0,1 \leqslant i \leqslant m$.

One can directly check that $M_{2}=\mathcal{F} \mathcal{L}\left(P_{2}\right)$. As we already know, $M_{2}$ is the final constraint submanifold, that is, $M_{2}=M_{f}$ with the usual notations. Observe that we can introduce local coordinates in $M_{2}$ as follows. Since $\chi_{i}=0$, for all $i$, we have

$$
\lambda^{i}=D^{i j} \mu_{j A} g^{A B} \hat{p}_{B}, \quad 1 \leqslant i \leqslant m
$$

Thus, we can take local coordinates $\left(q^{A}, \hat{p}_{A}\right)$ in $M_{2}$. More precisely, the mapping

$$
\left(q^{A}, \hat{p}_{A}\right) \mapsto\left(q^{A}, D^{i j} \mu_{j A} g^{A B} \hat{p}_{B}, \hat{p}_{A}, 0\right)
$$

defines $M_{2}$ as a submanifold of $T^{*} P$.

We summarize the above results in the following diagram:

$$
\begin{array}{ccc}
P_{1}=T P=T\left(Q \times \mathbb{R}^{m}\right) & \begin{array}{c}
\mathcal{F} \mathcal{L} \\
i_{1} \uparrow
\end{array} \\
P_{2} \equiv\left\langle\Phi_{i}=0\right\rangle & \begin{array}{c}
\mathcal{F} \mathcal{L}_{1} \\
\searrow
\end{array} & M_{1} \equiv\left\langle\hat{p}_{i}=0\right\rangle \\
& M_{\mathcal{L}_{2}} & \uparrow j_{2} \\
& M_{2} \equiv\left\langle\hat{p}_{i}=0, \chi_{i}=0\right\rangle .
\end{array}
$$

Remark V.2: Observe that $\omega_{2}=j_{2}^{*} \omega_{P}$ is in fact a symplectic form on $M_{2}$ since

$$
\operatorname{rank}\left(\omega_{2}\right)=2 n=\operatorname{dim} M_{2} .
$$

Then, we have that $\left(M_{2}, \omega_{2}, h_{2}\right)$ is a symplectic Hamiltonian system, where $h_{2}$ denotes the restriction of $h_{1}$ to $M_{2}$. In local coordinates,

$$
h_{2}=\frac{1}{2} g^{A B} \hat{p}_{B}\left(\hat{p}_{A}-D^{i k} \mu_{k C} \mu_{i A}{ }^{C D} \hat{p}_{D}\right)+U
$$

Let us denote by $\bar{M}=\mathcal{F} L(M)$ the submanifold of $T^{*} Q$ obtained by means of the Legendre transformation associated to $L$. Indeed, $\bar{M}$ is defined by the linear constraints $\mu_{i A} g^{A B} p_{B}$, where $\left(q^{A}, p_{A}\right)$ stand for the bundle coordinates in $T^{*} Q$. Notice that $\bar{M}$ is a vector subbundle of $T^{*} Q$ since $\mathcal{F} \mathcal{L}$ is a vector bundle isomorphism over $Q$. 
To end this section, we will investigate the relation between $M_{2}$ and $\bar{M}$, and will compare $\omega_{2}$ with $\omega_{Q}$, the canonical symplectic form on $T^{*} Q$.

Let $\gamma: M \times T R^{m} \rightarrow P_{2}$ be the global diffeomorphism between $M \times T \mathbb{R}^{m}$ and $P_{2}$, which is induced from the canonical diffeomorphism $T Q \times T \mathbb{R}^{m} \rightarrow T\left(Q \times \mathbb{R}^{m}\right)$. By means of $\gamma$, we define the global mapping

$$
\begin{aligned}
\delta: \quad \bar{M} \times \mathbb{R}^{m} & \rightarrow M_{2} \\
(\bar{y}, \lambda) & \mapsto \mathcal{F} \mathcal{L}\left(\gamma\left(\mathcal{F} L^{-1}(\bar{y}), \lambda, 0\right)\right) .
\end{aligned}
$$

In local coordinates we have

$$
\delta\left(q^{A}, p_{A}, \lambda^{i}\right)=\left(q^{A}, \lambda^{i}, p_{A}+\lambda^{i} \mu_{i A}, 0\right) .
$$

Proposition V.3: $\delta$ is a diffeomorphism.

Proof: Indeed, it is differentiable and its inverse is

$$
\begin{gathered}
M_{2} \rightarrow \bar{M} \times \mathbb{R}^{m}, \\
\left(q^{A}, \hat{p}_{A}\right) \mapsto\left(q^{A}, \hat{p}_{A}-\lambda^{i} \mu_{i A}, \lambda^{i}\right),
\end{gathered}
$$

where $\lambda^{i}=D^{i j} \mu_{j A} g^{A B} \hat{p}_{B}$. Obviously, $\delta^{-1}$ is differentiable, too.

Via $\delta$ one obtains that

$$
\delta^{*} \omega_{2}=\omega_{Q}-d\left(\lambda^{i} \mu_{i A}\right) \wedge d q^{A} .
$$

\section{THE SODE PROBLEM}

In this section we will discuss the problem of finding a vector field $\widetilde{\Gamma}$ satisfying the equations

$$
\begin{gathered}
\left(i_{\Gamma} \omega_{\mathcal{L}}=d E_{\mathcal{L}}\right)_{\mid \mathcal{S}}, \\
(S \widetilde{\Gamma}=\Delta)_{\mid \mathcal{S}},
\end{gathered}
$$

on some submanifold $\mathcal{S}$ of $P_{2}$. That is, we are looking for a solution satisfying the SODE condition, since our problem is variational and it requires second-order equations.

First of all, let us recall that points in the same fiber of $\mathcal{F} \mathcal{L}_{2}$ only differ one from each other in their components $\lambda^{i}$. Indeed, if $y_{0}$ is a point in $M_{2}$ with local coordinates $\left(q_{0}^{A}, \lambda_{0}^{i}, \hat{p}_{0 A}, 0\right)$ then we have

$$
\mathcal{F L}_{2}^{-1}\left(y_{0}\right)=\left\{\left(q_{0}^{A}, \lambda_{0}^{i}, g_{0}^{A B}\left(\hat{p}_{0 B}-\lambda_{0}^{i} \mu_{0 i B}\right), \dot{\lambda}^{i}\right) / \dot{\lambda}^{i} \in \mathbb{R}\right\} \subseteq P_{2} .
$$

This fact implies that, if

$$
\Gamma_{\mathcal{D}_{0}^{i}}=\dot{q}^{A} \frac{\partial}{\partial q^{A}}+\mathcal{B}^{i}(q, \lambda, \dot{q}) \frac{\partial}{\partial \lambda^{i}}+\mathcal{C}^{A}(q, \lambda, \dot{q}) \frac{\partial}{\partial \dot{q}^{A}}+\mathcal{D}_{0}^{i} \frac{\partial}{\partial \dot{\lambda}^{i}}
$$

is an arbitrary solution of Eq. (17), then it is projectable by $\mathcal{F} \mathcal{L}$ onto a vector field $\Gamma$ tangent to $M_{2}$ defined by

$$
\bar{\Gamma}(y)=\mathcal{F}_{*}\left(\Gamma_{D_{0}^{i}}(x)\right), \quad x \in \mathcal{F} \mathcal{L}^{-1}(y),
$$

since $\mathcal{B}^{i}$ and $\mathcal{C}^{A}$ do not depend on $\dot{\lambda}^{i}$.

Moreover, since $\bar{\Gamma}$ is such that $\left(i_{\bar{\Gamma}} \omega_{1}=d h_{1}\right)_{\mid M_{2}}$, we deduce 


$$
i_{\bar{\Gamma}} \omega_{2}=d h_{2},
$$

and $\bar{\Gamma}$ is the Hamiltonian vector field associated to $h_{2}$, i.e., $\bar{\Gamma}=\Gamma_{h_{2}}$. For each $y \in M_{2}$, with local coordinates $\left(q^{A}, \lambda^{i}, \hat{p}_{A}, 0\right)$ we have

$$
\begin{aligned}
\bar{\Gamma}(y)= & \mathcal{F} \mathcal{L}_{*}\left(\Gamma_{\mathcal{D}_{0}^{i}}(x)\right) \\
= & g^{A B}\left(\hat{p}_{B}-\lambda^{i} \mu_{i B}\right)\left(\frac{\partial}{\partial q^{A}}\right)_{y}+\mathcal{B}^{i}(x)\left(\frac{\partial}{\partial \lambda^{i}}\right)_{y} \\
& +\left(\left(\frac{\partial g_{A D}}{\partial q^{B}} \dot{q}^{D}+\lambda^{i} \frac{\partial \mu_{i A}}{\partial q^{B}}\right)_{y} g^{B C}\left(\hat{p}_{C}-\lambda^{i} \mu_{i C}\right)+\left(\mathcal{B}^{i} \mu_{i A}\right)(x)+\left(\mathcal{C}^{B} g_{A B}\right)(x)\right)\left(\frac{\partial}{\partial \hat{p}_{A}}\right)_{y} \\
= & g^{A B}\left(\hat{p}_{B}-\lambda^{i} \mu_{i B}\right)\left(\frac{\partial}{\partial q^{A}}\right)_{y}+\mathcal{B}^{i}(x)\left(\frac{\partial}{\partial \lambda^{i}}\right)_{y}+\frac{\partial \mathcal{L}}{\partial q^{A}}\left(\frac{\partial}{\partial \hat{p}_{A}}\right)_{y}
\end{aligned}
$$

where $x$ is an arbitrary point in $\mathcal{F} \mathcal{L}^{-1}(y)$.

Now, we define the mapping $s: M_{2} \rightarrow P_{2}$ by putting

$$
s(y)=s\left(q^{A}, \lambda^{i}, \hat{p}_{A}, 0\right)=\left(q^{A}, \lambda^{i}, g^{A B}\left(\hat{p}_{B}-\lambda^{i} \mu_{i B}\right), \mathcal{B}^{i}(x)\right), \quad y \in M_{2}, \quad x \in \mathcal{F} \mathcal{L}^{-1}(y),
$$

where $\lambda^{i}=D^{i j} \mu_{j A} g^{A B} \hat{p}_{B}$. It is not difficult to see that $s$ is well defined and that it does not depend on the choice of the local coordinates on $M_{2}$. In fact, one can define $s$ by taking the value of $\Gamma_{\mathcal{D}_{0}^{i}}$ at $x$ and then project the result by the canonical projection from $T P$ onto $P$ (see Refs. 31 and 33). Moreover, we have that $s(y) \in \mathcal{F} \mathcal{L}_{2}^{-1}(y)$, for each $y \in M_{2}$ so $s$ is a differentiable section of $\mathcal{F} \mathcal{L}_{2}$. Then, $\mathcal{S}=s\left(M_{2}\right) \subseteq P_{2}$ is a submanifold of $P_{2}$, and hence of $T P$ as well. Observe that on this submanifold, $\Gamma_{\mathcal{D}}$ satisfies the SODE condition: indeed, we have

$$
\left(S \Gamma_{\mathcal{D}}-\Delta\right)_{\mid \mathcal{S}}=\left(\left(\mathcal{B}^{i}-\dot{\lambda}^{i}\right) \frac{\partial}{\partial \lambda^{i}}\right)_{\mid \mathcal{S}}=0 .
$$

However, in general, one cannot ensure that $\Gamma_{\mathcal{D}}$ is tangent to $\mathcal{S}$.

This problem is solved by transporting the vector field $\bar{\Gamma}$ from $M_{2}$ to $\mathcal{S}$ by using the global diffeomorphism $s: M_{2} \rightarrow \mathcal{S}$, that is, we define

$$
\widetilde{\Gamma}=s_{*} \bar{\Gamma}
$$

Therefore, $\widetilde{\Gamma}$ will verify the SODE condition because of the form of $s$ and, in addition, the equation

$$
\left(i_{\Gamma} \omega_{\mathcal{L}}=d E_{\mathcal{L}}\right)_{\mid \mathcal{S}} .
$$

Next, we will obtain a local expression for $\widetilde{\Gamma}$. Let $x$ be a point in $\mathcal{S}$; since $s$ is injective, there is a unique point $y \in M_{2}$ such that $s(y)=x$. Then,

$$
\widetilde{\Gamma}(x)=s_{* y}(\bar{\Gamma}(y)) .
$$

As we know from the above discussion, $\dot{q}_{x}^{A}=g^{A B}\left(\hat{p}_{B}-\lambda^{i} \mu_{i B}\right)_{y}$ and $\dot{\lambda}_{x}^{i}=\mathcal{B}_{x}^{i}$, so that we have 


$$
\begin{aligned}
\widetilde{\Gamma}_{x}= & \dot{q}_{x}^{A}\left(\frac{\partial}{\partial q^{A}}\right)_{x}+\dot{\lambda}_{x}^{i}\left(\frac{\partial}{\partial \lambda^{i}}\right)_{x}+\left(\dot{q}_{x}^{A} \dot{q}_{x}^{D}\left(g_{C D} \frac{\partial g^{B C}}{\partial q^{A}}\right)_{x}-\dot{q}_{x}^{A} \lambda_{x}^{i} g_{x}^{B C}\left(\frac{\partial \mu_{i C}}{\partial q^{A}}\right)_{x}-\left(\dot{\lambda}^{i} g^{B C} \mu_{i C}\right)_{x}\right. \\
& \left.+g_{x}^{B A}\left[\left(\frac{\partial g_{E A}}{\partial q^{D}} \dot{q}^{E}+\lambda^{i} \frac{\partial \mu_{i A}}{\partial q^{D}}\right)_{x} \dot{q}_{x}^{D}+\left(\dot{\lambda}^{i} \mu_{i A}\right)_{x}+\left(\mathcal{C}^{D} g_{A D}\right)_{x}\right]\right)\left(\frac{\partial}{\partial \dot{q}^{B}}\right)_{x} \\
& +\left(\dot{q}_{x}^{A}\left(\frac{\partial \mathcal{B}^{i}}{\partial q^{A}}\right)_{x}+\dot{\lambda}_{x}^{j}\left(\frac{\partial \mathcal{B}^{i}}{\partial \lambda^{j}}\right)_{x}+\frac{\partial \mathcal{L}}{\partial q^{A}}\left(\frac{\partial \mathcal{B}^{i}}{\partial \hat{p}_{A}}\right)_{x}\right)\left(\frac{\partial}{\partial \dot{\lambda}^{i}}\right)_{x} .
\end{aligned}
$$

This expression can be simplified as follows:

$$
\begin{aligned}
\widetilde{\Gamma}_{x} & =\dot{q}_{x}^{A}\left(\frac{\partial}{\partial q^{A}}\right)_{x}+\dot{\lambda}_{x}^{i}\left(\frac{\partial}{\partial \lambda^{i}}\right)_{x}+\mathcal{C}_{x}^{B}\left(\frac{\partial}{\partial \dot{q}^{B}}\right)_{x}+\left(\dot{q}_{x}^{A}\left(\frac{\partial \mathcal{B}^{i}}{\partial q^{A}}\right)_{x}+\dot{\lambda}_{x}^{j}\left(\frac{\partial \mathcal{B}^{i}}{\partial \lambda^{j}}\right)_{x}+\dot{\hat{p}}_{A}\left(\frac{\partial \mathcal{B}^{i}}{\partial \hat{p}_{A}}\right)_{x}\right)\left(\frac{\partial}{\partial \dot{\lambda}^{i}}\right)_{x} \\
& =\dot{q}_{x}^{A}\left(\frac{\partial}{\partial q^{A}}\right)_{x}+\dot{\lambda}_{x}^{i}\left(\frac{\partial}{\partial \lambda^{i}}\right)_{x}+\mathcal{C}_{x}^{B}\left(\frac{\partial}{\partial \dot{q}^{B}}\right)_{x}+\dot{\mathcal{B}}_{x}^{i}\left(\frac{\partial}{\partial \dot{\lambda}^{i}}\right),
\end{aligned}
$$

taking into account that

$$
\dot{q}^{A} g_{C D} \dot{q}^{D} \frac{\partial g^{B C}}{\partial q^{A}}+\dot{q}^{D} \dot{q}^{E} g^{B A} \frac{\partial g_{E A}}{\partial q^{D}}=\dot{q}^{D} \dot{q}^{E} \frac{\partial}{\partial q^{D}}\left(g^{B C} g_{C E}\right)=0 .
$$

Remark VI.1: We have obtained a vector field $\bar{\Gamma}$ on $M_{2}$, and a vector field $\widetilde{\Gamma}$ on $\mathcal{S}$, both vector fields solving the dynamics of the singular Lagrangian $\mathcal{L}$. It should be noticed that, since the equations of motion for $\mathcal{L}$ are the same as the equations of motion for the vakonomic problem, we have obtained a sort of reduction of the latter problem. Indeed, the integral curves of $\bar{\Gamma}$ (or equivalently, of $\widetilde{\Gamma}$ ) give the vakonomic dynamics. But $M_{2}$ (or, if we want, $\mathcal{S}$ ) has dimension $2 n$ and we have started with a state system $T P$ with dimension $2 n+2 m$.

Recall that we have proved $\bar{\Gamma}=\Gamma_{h_{2}}$. In addition, the vector field $\widetilde{\Gamma}$ on $\mathcal{S}$ is also a Hamiltonian vector field. In fact, $\widetilde{\Gamma}$ is the Hamiltonian vector field corresponding to the restriction of $E_{L}$ and with respect to the restriction of $\omega_{\mathcal{L}}$ to $\mathcal{S}$. Both Hamiltonian vector fields are related by the symplectomorphism $s$.

\section{CLASSIFICATION OF THE CONSTRAINTS ACCORDING TO DIRAC}

The application of the Dirac-Bergmann-Gotay-Nester algorithm has produced the following constraints:

(i) the primary constraints, $\hat{p}_{j}=0,1 \leqslant j \leqslant m$,

(ii) and the secondary constraints, $\chi_{j}=0,1 \leqslant j \leqslant m$,

which together define the final constraint submanifold $M_{2}$.

In according with Dirac's terminology, ${ }^{29}$ the constraints can be classified into first class and second class constraints. Let us recall that a constraint is said to be first class if its brackets with all the other constraints vanish; otherwise, it is said to be second class.

Here the bracket is the canonical one provided by the canonical symplectic form $\omega_{P}$ on $T^{*} P$,

$$
\{\bar{f}, \bar{g}\}=\frac{\partial \bar{f}}{\partial q^{A}} \frac{\partial \bar{g}}{\partial \hat{p}_{A}}+\frac{\partial \bar{f}}{\partial \lambda^{i}} \frac{\partial \bar{g}}{\partial \hat{p}_{i}}-\frac{\partial \bar{f}}{\partial \hat{p}_{i}} \frac{\partial \bar{g}}{\partial \lambda^{i}}-\frac{\partial \bar{f}}{\partial \hat{p}_{A}} \frac{\partial \bar{g}}{\partial q^{A}},
$$


for all pair of functions $\bar{f}, \bar{g}: T^{*} P \rightarrow \mathbb{R}$.

We construct the matrix $C=\left(C_{\alpha \beta}\right)$, with $C_{\alpha \beta}=\left\{\varphi_{\alpha}, \varphi_{\beta}\right\}$, where $1 \leqslant \alpha \leqslant 2 m$ and $\varphi_{\alpha}=\hat{p}_{\alpha}$ for $1 \leqslant \alpha \leqslant m$ and $\varphi_{\alpha}=\chi_{\alpha-m}$ if $m+1 \leqslant \alpha \leqslant 2 m$. Then we have

$$
\left(C_{\alpha \beta}\right)=\left(\begin{array}{ll}
\left\{\hat{p}_{i}, \hat{p}_{j}\right\} & \left\{\hat{p}_{i}, \chi_{j}\right\} \\
\left\{\chi_{i}, \hat{p}_{j}\right\} & \left\{\chi_{i}, \chi_{j}\right\}
\end{array}\right)=\left(\begin{array}{cc}
0 & D_{i j} \\
-D_{i j} & N_{i j}
\end{array}\right),
$$

with

$$
N_{i j}=\left\{\chi_{i}, \chi_{j}\right\}=\hat{p}_{C} g^{A B}\left(\mu_{j A} \frac{\partial\left(\mu_{i D} g^{C D}\right)}{\partial q^{B}}-\mu_{i A} \frac{\partial\left(\mu_{j D} g^{C D}\right)}{\partial q^{B}}\right)+g^{A B} \lambda^{k}\left(\mu_{i A} \frac{\partial D_{k j}}{\partial q^{B}}-\mu_{j A} \frac{\partial D_{k i}}{\partial q^{B}}\right) .
$$

A straightforward computation shows that the matrix $C$ is invertible with inverse

$$
C^{-1}=\left(C^{\alpha \beta}\right)=\left(\begin{array}{cc}
D^{-1} N D^{-1} & -D^{-1} \\
D^{-1} & 0
\end{array}\right) .
$$

Therefore, all the constraints are second class.

Thus, the Dirac bracket is

$$
\{\bar{f}, \bar{g}\}_{D}=\{\bar{f}, \bar{g}\}-\left\{\bar{f}, \varphi_{\alpha}\right\} C^{\alpha \beta}\left\{\varphi_{\beta}, \bar{g}\right\},
$$

for all pair of functions $\bar{f}$ and $\bar{g}$ on $T^{*} P$.

An important observation is the following. Since the constraints become Casimir functions with respect to the Dirac bracket, then it can be restricted to $M_{2}$. Indeed, for all pairs of functions $f, g \in C^{\infty}\left(M_{2}\right)$ the bracket $\{\bar{f}, \bar{g}\}_{D \mid M_{2}}$ does not depend on the choice of the extensions $\bar{f}, \bar{g}$ to $T^{*} P$. Consequently, we will denote $\{f, g\}^{*}=\{\bar{f}, \bar{g}\}_{D \mid M_{2}}$.

As Dirac proved, the bracket $\{,\}_{D}$ provides the evolution of any observable, that is,

$$
\dot{\bar{f}}=\{\bar{f}, \bar{h}\}_{D},
$$

for some convenient extension $\bar{h}$ of the projected Hamiltonian $h_{1} \in C^{\infty}\left(M_{1}\right)$. In particular, $\left\{f, h_{2}\right\}^{*}$ gives the evolution of $f: M_{2} \rightarrow \mathbb{R}$.

As we have noticed in Sec. V, $\left(M_{2}, \omega_{2}\right)$ is a symplectic submanifold of $T^{*} P$. Let us denote by $\{,\}_{M_{2}}$ the Poisson bracket induced by $\omega_{2}$. We are interested in knowing which is the relation between both brackets, $\{,\}^{*}$ and $\{,\}_{M_{2}}$. This is solved in the following.

Proposition VII.1: The bracket $\{,\}^{*}$ coincides with $\{,\}_{M_{2}}$, that is, we have that

$$
\{f, g\}^{*}=\{f, g\}_{M_{2}},
$$

for all $f, g \in C^{\infty}\left(M_{2}\right)$.

Proof: As $\left(M_{2}, \omega_{2}\right)$ is a symplectic submanifold of $T^{*} P$, we have the following decomposition:

$$
T_{M_{2}}\left(T^{*} P\right)=T M_{2} \oplus T M_{2}^{\perp},
$$

with associated projectors

$$
\begin{gathered}
\mathcal{P}: T_{M_{2}}\left(T^{*} P\right) \rightarrow T M_{2}, \\
\mathcal{Q}: T_{M_{2}}\left(T^{*} P\right) \rightarrow T M_{2}^{\perp} .
\end{gathered}
$$


It is proved in Ref. 34 that our Dirac bracket is precisely

$$
\{\bar{f}, \bar{g}\}_{D}=\omega_{P}\left(\mathcal{P}\left(X_{f}^{-}\right), \mathcal{P}\left(X_{\bar{g}}\right)\right)
$$

for $\bar{f}, \bar{g} \in C^{\infty}\left(T^{*} P\right)$. Let us denote by $Y_{f}$ the Hamiltonian vector field on $M_{2}$ associated with a function $f: M_{2} \rightarrow \mathbb{R}$ with respect to $\omega_{2}$. A careful computation shows that $j_{2 *} Y_{f}=\mathcal{P}\left(X_{\bar{f}}^{-}\right)$, where $\bar{f}$ is an extension to $T^{*} P$ of $f \in C^{\infty}\left(M_{2}\right)$. Consequently, we have

$$
\{f, g\}^{*}=\omega_{P}\left(\mathcal{P}\left(X_{\bar{f}}^{-}\right), \mathcal{P}\left(X_{\bar{g}}\right)\right)=\omega_{P}\left(j_{2 *} Y_{f}, j_{2 *} Y_{g}\right)=\omega_{2}\left(Y_{f}, Y_{g}\right)=\{f, g\}_{M_{2}}
$$

If we denote by $\pi: \bar{M} \times \mathbb{R}^{m} \rightarrow \bar{M}$ the canonical projection, we can define a Poisson bracket along $\widetilde{\pi}=\pi \circ \delta^{-1}$ as follows:

$$
\{f, g\}_{\mathrm{vak}}=\{f \circ \widetilde{\pi}, g \circ \widetilde{\pi}\}^{*},
$$

which is a function defined on $M_{2}$. Therefore, we have a bracket

$$
\begin{aligned}
& \{,\}_{\text {vak }}: \quad C^{\infty}(\bar{M}) \times C^{\infty}(\bar{M}) \quad \longrightarrow \quad C^{\infty}\left(M_{2}\right) \\
& (f, g) \longrightarrow\{f, g\}_{\text {vak }}
\end{aligned}
$$

which is in fact a bracket along $\widetilde{\pi}$. This bracket $\{,\}_{\text {vak }}$ enjoys similar properties to those of ordinary Poisson brackets.

Definition VII.2: The bracket \{\}$_{\mathrm{vak}}$ on $\bar{M}$ along $\widetilde{\pi}$ will be called the vakonomic bracket. The vakonomic bracket produces a function on $M_{2}$ from two functions defined on $\bar{M}$, since we need to specify the corresponding Lagrange multipliers $\lambda^{i}$ in the equations by means of the above-mentioned diffeomorphism between $M_{2}$ and $\bar{M} \times \mathbb{R}^{m}$.

A careful computation shows that, in local coordinates, the expression for the vakonomic bracket is

$$
\{f, g\}_{\mathrm{vak}}=\{f \circ \widetilde{\pi}, g \circ \widetilde{\pi}\}^{*}=\frac{\partial(f \circ \widetilde{\pi})}{\partial q^{A}} \frac{\partial(g \circ \widetilde{\pi})}{\partial \hat{p}_{A}}-\frac{\partial(f \circ \widetilde{\pi})}{\partial \hat{p}_{A}} \frac{\partial(g \circ \widetilde{\pi})}{\partial q^{A}}+\frac{\partial \bar{f}}{\partial \lambda^{i}} D^{i k} N_{j l} D^{l j} \frac{\partial \bar{g}}{\partial \lambda^{j}},
$$

where $\bar{f}, \bar{g} \in C^{\infty}\left(T^{*} P\right)$ are arbitrary extensions of $f \circ \widetilde{\pi}$ and $g \circ \widetilde{\pi}$, respectively.

Moreover, if $\bar{\Gamma}$ is the "reduced" vakonomic vector field on $M_{2}$, then, for any $f: \bar{M} \rightarrow \mathbb{R}$, we have

$$
\left\{f, H_{\mid \bar{M}}\right\}_{\mathrm{vak}}=\left\{f \circ \widetilde{\pi}, H_{\mid \bar{M}^{\circ}} \widetilde{\pi}\right\}^{*}=\bar{\Gamma}(f \circ \widetilde{\pi}) \equiv \dot{f},
$$

where $H: T^{*} Q \rightarrow \mathbb{R}$ is the Hamiltonian defined by $E_{L}$, that is, $H \circ \mathcal{F} L=E_{L}$.

Remark VII.3: It should be noticed that $M_{2}$ has a vector bundle structure over $\bar{M}$ with rank $m$. Indeed, it is a vector subbundle of $p r_{1}: T^{*} P \equiv T^{*} Q \times \mathbb{R}^{2 m} \rightarrow T^{*} Q$, that is,

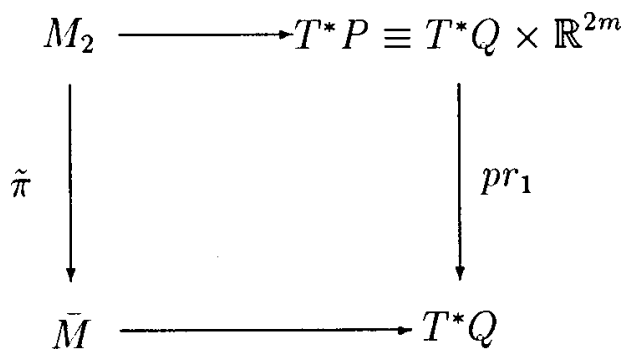


In this way, a vakonomic motion $(q(t), \lambda(t))$ in $M_{2}$ can be viewed as a motion in the total space of that vector bundle, with base components $q(t)$ in $\bar{M}$ and fiber components $\lambda(t)$ in $\mathbb{R}^{m}$. Roughly speaking, the Lagrange multipliers can be considered as a sort of internal variables in addition to position variables.

Example VII.4: The vertical rolling disk. Let us consider the following problem for a disk of radius $R$ and unit mass $m=1$ which rolls on a horizontal plane.

The configuration space for this system can be identified with $Q=\mathbb{R}^{2} \times S^{1} \times S^{1}$. By $(x, y)$ $\in \mathbb{R}^{2}$ we denote the coordinates of the point of contact of the disk with the plane and $(\theta, \varphi)$ $\in S^{1} \times S^{1}$ give, respectively, the angle between the disk and the $x$ axis, and the angle of rotation between a fixed diameter in the disk and the $y$ axis.

Given $q_{0}, q_{1} \in Q$, i.e., initial and final position variables, we want to find the trajectories of the disk connecting such points that minimize the energy expenditure. Of course, we want the disk to roll without slipping. This situation can be seen as an optimal control problem. ${ }^{36} \mathrm{~A}$ problem of optimal control is described by the following data: a configuration space $B$ giving the states variables of the system, a fiber bundle $\pi: N \rightarrow B$ whose fibers describe the control variables, a vector field $Y: N \rightarrow T B$ along the projection $\pi$, and a "Lagrangian" function $L: N \rightarrow \mathbb{R}$. Now the solutions of the optimal control problem will be those paths $\gamma: I \rightarrow N$ such that $\pi^{\circ} \gamma$ has fixed end points, which extremize the action

$$
\int_{\gamma} L(\gamma(t)) d t
$$

and satisfy the differential equation

$$
\frac{d}{d t}(\pi \circ \gamma)=Y \circ \gamma,
$$

which rules the evolution of the state variables.

It is easy to show that this is indeed a vakonomic problem on the manifold $N$. The constraint submanifold $M \subset T N$, given by the above-mentioned differential equation is

$$
M=\left\{v_{n} \in T N / \pi_{*}\left(v_{n}\right)=Y(n)\right\} .
$$

In the problem under consideration, we identify $B=Q, N=T Q$, and $\pi: T Q \rightarrow Q$ as the natural projection $\tau_{Q}$. The Lagrangian $L: T Q \rightarrow \mathbb{R}$ is given by

$$
L=\frac{1}{2}\left(\dot{x}^{2}+\dot{y}^{2}+I_{1} \dot{\theta}^{2}+I_{1} \dot{\varphi}^{2}\right),
$$

with $I_{1}, I_{2}$ the moments of inertia (notice that the potential energy is not included since it is constant). The vector field along $\tau_{Q}$ is

$$
\begin{aligned}
& \begin{array}{llll}
Y: & T Q & \longrightarrow & T Q
\end{array} \\
& \left(x, y, \theta, \varphi, d_{1}, d_{2}, d_{3}, d_{4}\right) \quad \rightarrow \quad\left(x, y, \theta, \varphi, R \cos \theta d_{4}, R \sin \theta d_{4}, d_{3}, d_{4}\right) .
\end{aligned}
$$

Notice that $Y$ is simply a tensor $(1,1)$ on the manifold $Q$.

In fact, in this framework, we are considering the velocities as the "control" variables. Solving this optimal control problem is precisely the same as considering the vakonomic problem associated with the vertical rolling disk for the extended Lagrangian $\mathcal{L}: T\left(Q \times \mathbb{R}^{2}\right) \rightarrow \mathbb{R}$,

$$
\mathcal{L}=L+\lambda \phi+\mu \psi \text {. }
$$

where

$$
\phi=\dot{x} \sin \theta-\dot{y} \cos \theta,
$$




$$
\psi=\dot{x} \cos \theta+\dot{y} \sin \theta-R \dot{\varphi}
$$

are the constraint functions determining $M$. Note that we have chosen a linear combination of the usual constraints

$$
\begin{aligned}
& \bar{\phi}=\dot{x}-R \dot{\varphi} \cos \theta, \\
& \bar{\psi}=\dot{y}-R \dot{\varphi} \sin \theta .
\end{aligned}
$$

In Sec. VIII, we will discuss how this change of constraints affects the final result. In addition, as is stated in Refs. 8 and 24, the vakonomic solutions for this problem are also solutions of the nonholonomic problem if the initial conditions for the Lagrange multipliers are properly chosen.

We have that

$$
\begin{aligned}
\omega_{\mathcal{L}}= & d x \wedge d \dot{x}+\sin \theta d x \wedge d \lambda+\cos \theta d x \wedge d \mu+(\lambda \cos \theta-\mu \sin \theta) d x \wedge d \theta+d y \wedge d \dot{y}-\cos \theta d y \wedge d \lambda \\
& +\sin \theta d y \wedge d \mu+(\lambda \sin \theta+\mu \cos \theta) d y \wedge d \theta+I_{1} d \theta \wedge d \dot{\theta}+I_{2} d \varphi \wedge d \dot{\varphi}-R d \varphi \wedge d \mu
\end{aligned}
$$

is the Poincare-Cartan two-form in local coordinates.

The final constraint submanifold is

$$
P_{2}=\left\{(x, y, \theta, \varphi, \lambda, \mu, \dot{x}, \dot{y}, \dot{\theta}, \dot{\varphi}, \dot{\lambda}, \dot{\mu}) \in T\left(Q \times \mathrm{R}^{2}\right) / \phi=0, \psi=0\right\}
$$

Let $\Gamma$ be a general solution of equation $i_{\Gamma} \omega_{\mathcal{L}}=d E_{L}$ and tangent to $P_{2}$. In local coordinates, we have

$$
\Gamma=\dot{x} \frac{\partial}{\partial x}+\dot{y} \frac{\partial}{\partial y}+\dot{\theta} \frac{\partial}{\partial \theta}+\dot{\varphi} \frac{\partial}{\partial \varphi}+\mathcal{B}_{\lambda} \frac{\partial}{\partial \lambda}+\mathcal{B}_{\mu} \frac{\partial}{\partial \mu}+\mathcal{C}_{x} \frac{\partial}{\partial \dot{x}}+\mathcal{C}_{y} \frac{\partial}{\partial \dot{y}}+\mathcal{C}_{\theta} \frac{\partial}{\partial \dot{\theta}}+\mathcal{C}_{\varphi} \frac{\partial}{\partial \dot{\varphi}}+\mathcal{D}_{\lambda} \frac{\partial}{\partial \dot{\lambda}}+\mathcal{D}_{\mu} \frac{\partial}{\partial \dot{\mu}}
$$

The coefficients satisfy the following equations:

$$
\begin{gathered}
\mathcal{C}_{x}=-\mathcal{B}_{\lambda} \sin \theta-\mathcal{B}_{\mu} \cos \theta-\dot{\theta}(\lambda \cos \theta-\mu \sin \theta), \\
\mathcal{C}_{y}=\mathcal{B}_{\lambda} \cos \theta-\mathcal{B}_{\mu} \sin \theta-\dot{\theta}(\lambda \sin \theta+\mu \cos \theta), \\
\mathcal{C}_{\theta}=\frac{R}{I_{1}} \lambda \dot{\varphi}, \\
\mathcal{C}_{\varphi}=\frac{R}{I_{2}} \mathcal{B}_{\mu},
\end{gathered}
$$

and the tangency conditions

$$
\begin{aligned}
\Gamma(\phi) & =\mathcal{C}_{x} \sin \theta-\mathcal{C}_{y} \cos \theta+R \dot{\theta} \dot{\varphi}=0, \\
\Gamma(\psi) & =\mathcal{C}_{x} \cos \theta+\mathcal{C}_{y} \sin \theta-R \mathcal{C}_{\varphi}=0
\end{aligned}
$$

Therefore, we get

$$
\left(\begin{array}{cc}
1 & 0 \\
0 & \left(1+\frac{R^{2}}{I_{2}}\right)
\end{array}\right)\left(\begin{array}{l}
\mathcal{B}_{\lambda} \\
\mathcal{B}_{\mu}
\end{array}\right)=\left(\begin{array}{c}
\dot{\theta}(R \dot{\varphi}+\mu) \\
-\lambda \dot{\theta}
\end{array}\right),
$$

which leads to 


$$
\begin{gathered}
\mathcal{B}_{\lambda}=R \dot{\theta} \dot{\varphi}+\mu \dot{\theta}, \\
\mathcal{B}_{\mu}=a \lambda \dot{\theta},
\end{gathered}
$$

where $a=-\left(1+\left(R^{2} / I_{2}\right)\right)^{-1}$. In turn, the expressions for the other coefficients of $\Gamma$ become

$$
\begin{gathered}
\mathcal{C}_{x}=-(1+a) \lambda \dot{\theta} \cos \theta-R \dot{\varphi} \dot{\theta} \sin \theta, \\
\mathcal{C}_{y}=-(1+a) \lambda \dot{\theta} \sin \theta+R \dot{\varphi} \dot{\theta} \cos \theta, \\
\mathcal{C}_{\theta}=\frac{R}{I_{1}} \lambda \dot{\varphi}, \\
\mathcal{C}_{\varphi}=\frac{R a}{I_{2}} \lambda \dot{\theta} .
\end{gathered}
$$

Continuing with the described process, we have that the submanifold $\mathcal{S}$ is given by

$$
\mathcal{S}=\left\{(x, y, \theta, \varphi, \lambda, \mu, \dot{x}, \dot{y}, \dot{\theta}, \dot{\varphi}, \dot{\lambda}, \dot{\mu}) \in T\left(Q \times \mathbb{R}^{2}\right) / \phi=0, \psi=0, \mathcal{B}_{\lambda}=\dot{\lambda}, \mathcal{B}_{\mu}=\dot{\mu}\right\},
$$

and $\widetilde{\Gamma}$ is

$$
\widetilde{\Gamma}=\dot{x} \frac{\partial}{\partial x}+\dot{y} \frac{\partial}{\partial y}+\dot{\theta} \frac{\partial}{\partial \theta}+\dot{\varphi} \frac{\partial}{\partial \varphi}+\dot{\lambda} \frac{\partial}{\partial \lambda}+\dot{\mu} \frac{\partial}{\partial \mu}+\mathcal{C}_{x} \frac{\partial}{\partial \dot{x}}+\mathcal{C}_{y} \frac{\partial}{\partial \dot{y}}+\mathcal{C}_{\theta} \frac{\partial}{\partial \dot{\theta}}+\mathcal{C}_{\varphi} \frac{\partial}{\partial \dot{\varphi}}+\mathcal{D}_{\lambda} \frac{\partial}{\partial \dot{\lambda}}+\mathcal{D}_{\mu} \frac{\partial}{\partial \dot{\mu}}
$$

with

$$
\begin{aligned}
& \mathcal{D}_{\lambda}=-\lambda \dot{\theta}^{2}+\frac{R^{2}}{I_{1}} \lambda \dot{\varphi}^{2}+\frac{R}{I_{1}} \mu \lambda \dot{\varphi}, \\
& \mathcal{D}_{\mu}=a R \dot{\varphi} \dot{\theta}^{2}+a \mu \dot{\theta}^{2}+\frac{a R}{I_{1}} \lambda^{2} \dot{\varphi} .
\end{aligned}
$$

Observe that the equations for the Lagrange multipliers

$$
\begin{gathered}
\dot{\lambda}=\dot{\theta}(R \dot{\varphi}+\dot{\mu}), \\
\dot{\mu}=a \lambda \dot{\theta},
\end{gathered}
$$

can be integrated to give

$$
\begin{gathered}
\lambda=A \sin \theta-B \cos \theta, \\
\mu=A \cos \theta+B \sin \theta-R \dot{\varphi},
\end{gathered}
$$

where $A$ and $B$ are constants which depend on the initial conditions $\lambda(0), \mu(0)$. This allows us to project $\widetilde{\Gamma}_{(A, B)}$ to a vector field $X_{(A, B)}$ on $M$ giving different vakonomic solutions for each choice of $(A, B)$. In particular

$$
X_{(0,0)}=\dot{x} \frac{\partial}{\partial x}+\dot{y} \frac{\partial}{\partial y}+\dot{\theta} \frac{\partial}{\partial \theta}+\dot{\varphi} \frac{\partial}{\partial \varphi}-R \dot{\varphi} \dot{\theta} \sin \theta \frac{\partial}{\partial \dot{x}}+R \dot{\varphi} \dot{\theta} \cos \theta \frac{\partial}{\partial \dot{y}}
$$


is just the nonholonomic vector field, $\Gamma_{L, M}$, corresponding to the vertical rolling disk (see the discussion at the end of Sec. IV).

Now, the Legendre transformation $\mathcal{F} \mathcal{L}: T\left(Q \times \mathbb{R}^{2}\right) \rightarrow T^{*}\left(Q \times \mathbb{R}^{2}\right)$ is given by

$$
\mathcal{F} \mathcal{L}(x, y, \theta, \varphi, \lambda, \mu, \dot{x}, \dot{y}, \dot{\theta}, \dot{\varphi}, \dot{\lambda}, \dot{\mu})=\left(x, y, \theta, \varphi, \lambda, \mu, \hat{p}_{x}, \hat{p}_{y}, \hat{p}_{\theta}, \hat{p}_{\varphi}, \hat{p}_{\lambda}, \hat{p}_{\mu}\right),
$$

where

$$
\begin{gathered}
\hat{p}_{x}=\dot{x}+\lambda \sin \theta+\mu \cos \theta, \\
\hat{p}_{y}=\dot{y}-\lambda \cos \theta+\mu \sin \theta, \\
\hat{p}_{\theta}=I_{1} \dot{\theta}, \\
\hat{p}_{\varphi}=I_{2} \dot{\varphi}-R \mu, \\
\hat{p}_{\lambda}=0, \\
\hat{p}_{\mu}=0 .
\end{gathered}
$$

So the presymplectic system $\left(M_{1}, \omega_{1}, h_{1}\right)$ becomes

$$
\begin{gathered}
M_{1}=\mathcal{F} \mathcal{L}\left(T\left(Q \times \mathbb{R}^{2}\right)\right) \equiv \mathbb{R}^{10}, \\
\omega_{1}=d x \wedge d \hat{p}_{x}+d y \wedge d \hat{p}_{y}+d \theta \wedge d \hat{p}_{\theta}+d \varphi \wedge \hat{p}_{\varphi}, \\
h_{1}=\frac{1}{2}\left(\left(\hat{p}_{x}-\lambda \sin \theta-\mu \cos \theta\right)^{2}+\left(\hat{p}_{y}+\lambda \cos \theta-\mu \sin \theta\right)^{2}+\frac{1}{I_{1}} \hat{p}_{\theta}^{2}+\frac{1}{I_{2}}\left(\hat{p}_{\varphi}+R \mu\right)^{2}\right) .
\end{gathered}
$$

Applying Gotay-Nester's algorithm we get the secondary constraints

$$
\begin{gathered}
\chi_{\lambda}=-\lambda-\hat{p}_{y} \cos \theta+\hat{p}_{x} \sin \theta, \\
\chi_{\mu}=a^{-1} \mu+\hat{p}_{y} \sin \theta+\hat{p}_{x} \cos \theta-\frac{R}{I_{2}} \hat{p}_{\varphi},
\end{gathered}
$$

through which we obtain the symplectic Hamiltonian system $\left(M_{2}, \omega_{2}, h_{2}\right)$

$$
\begin{gathered}
M_{2}=\mathcal{F} \mathcal{L}\left(P_{2}\right) \equiv \mathbb{R}^{8}, \\
\omega_{2}=d x \wedge d \hat{p}_{x}+d y \wedge d \hat{p}_{y}+d \theta \wedge d \hat{p}_{\theta}+d \varphi \wedge d \hat{p}_{\varphi}, \\
h_{2}=\frac{1}{2}\left((1+a) \cos ^{2} \theta \hat{p}_{x}^{2}+(1+a) \sin ^{2} \theta \hat{p}_{y}^{2}+\frac{1}{I_{1}} \hat{p}_{\theta}^{2}-\frac{a}{I_{2}} \hat{p}_{\varphi}^{2}\right. \\
\left.+(1+a) \sin 2 \theta \hat{p}_{x} \hat{p}_{y}-2 \frac{R a}{I_{2}} \cos \theta \hat{p}_{x} \hat{p}_{\varphi}-2 \frac{R a}{I_{2}} \sin \theta \hat{p}_{y} \hat{p}_{\varphi}\right) .
\end{gathered}
$$

As we have said, the natural bracket associated with the two-form $\omega_{2}$ allows us to construct the vakonomic bracket. This is, for any $f, g: \bar{M} \rightarrow \mathrm{R}$ we have

$$
\{f, g\}_{\mathrm{vak}}=\{f \circ \widetilde{\pi}, g \circ \widetilde{\pi}\}_{M_{2}},
$$

where $\widetilde{\pi}: M_{2} \rightarrow \bar{M}$ is 


$$
\begin{gathered}
\tilde{\pi}(z)=\left(x, y, \theta, \varphi,(1+a) \cos ^{2} \theta \hat{p}_{x}+(1+a) \sin \theta \cos \theta \hat{p}_{y}-\frac{R a}{I_{2}} \cos \theta \hat{p}_{\varphi}\right. \\
\left.(1+a) \sin \theta \cos \theta \hat{p}_{x}+(1+a) \sin ^{2} \theta \hat{p}_{y}-\frac{R a}{I_{2}} \sin \theta \hat{p}_{\varphi}, \quad \hat{p}_{\theta},-a\left(R \cos \theta \hat{p}_{x}+R \sin \theta \hat{p}_{y}+\hat{p}_{\varphi}\right)\right) .
\end{gathered}
$$

If $H_{\mid \bar{M}}$ is the restriction of $H$ to $\bar{M}$, since $H_{\mid \bar{M}^{\circ}} \widetilde{\pi}=h_{2}$ we have

$$
\begin{aligned}
\left\{f, H_{\mid \bar{M}}\right\}_{\mathrm{vak}}= & \left\{f \circ \widetilde{\pi}, h_{2}\right\}^{*} \\
= & \frac{\partial(f \circ \widetilde{\pi})}{\partial x}\left((1+a) \cos ^{2} \theta \hat{p}_{x}+(1+a) \sin \theta \cos \theta \hat{p}_{y}-\frac{2 R a}{I_{2}} \cos \theta \hat{p}_{\phi}\right) \\
& +\frac{\partial(f \circ \widetilde{\pi})}{\partial y}\left((1+a) \sin ^{2} \theta \hat{p}_{y}+(1+a) \sin \theta \cos \theta \hat{p}_{x}-\frac{2 R a}{I_{2}} \sin \theta \hat{p}_{\phi}\right) \\
& +\frac{\partial(f \circ \widetilde{\pi})}{\partial \theta} \frac{\hat{p}_{\theta}}{I_{1}}-\frac{\partial(f \circ \widetilde{\pi})}{\partial \varphi}\left(\frac{a}{I_{2}} \hat{p}_{\phi}+\frac{R a}{I_{2}} \cos \theta \hat{p}_{x}-\frac{R a}{I_{2}} \sin \theta \hat{p}_{y}\right) \\
& -\frac{1}{2} \frac{\partial(f \circ \widetilde{\pi})}{\partial \hat{p}_{\theta}}\left(-(1+a) \sin 2 \theta \hat{p}_{x}^{2}+(1+a) \sin 2 \theta \hat{p}_{y}^{2}+(1+a) 2 \cos 2 \theta \hat{p}_{x} \hat{p}_{y}\right. \\
& \left.+\frac{2 R a}{I_{2}} \sin \theta \hat{p}_{x} \hat{p}_{\varphi}-\frac{2 R a}{I_{2}} \cos \theta \hat{p}_{y} \hat{p}_{\varphi}\right) .
\end{aligned}
$$

\section{CONSISTENCY OF THE LOCAL CONSTRUCTION}

In the previous sections we have assumed that the constraint functions $\Phi_{i}$ were globally defined on the whole of $T Q$. Under this assumption, we have defined the extended Lagrangian $\mathcal{L}$ on TP and, by means of the constraint algorithm, we have obtained an equivalent description of vakonomic dynamics in terms of the vector fields $\widetilde{\Gamma}$ and $\bar{\Gamma}$, on $S$ and $M_{2}$, respectively. An alternative description was provided by the bracket $\{,\}_{\text {vak }}$.

In this section, we will discuss the validity of the above results when a change of constraints or a change of local coordinates is performed. We accomplish the two tasks at the same time. Suppose that $V$ and $\bar{V}$ are two coordinate neighborhoods in the configuration manifold $Q$ such that $V \cap \bar{V} \neq \varnothing$, and denote by $\left(q^{A}\right)$ and $\left(\bar{q}^{A}\right)$ the corresponding coordinate functions. Let

$$
\begin{gathered}
\Phi_{i}: T V \rightarrow \mathbb{R}, \quad \Phi_{i}=\mu_{i A} \dot{q}^{A}, \\
\bar{\Phi}_{j}: T \bar{V} \rightarrow \mathbb{R}, \quad \bar{\Phi}_{j}=\bar{\mu}_{j B} \dot{\bar{q}}^{B},
\end{gathered}
$$

be two sets of constraints defining $M \cap T V$ and $M \cap T \bar{V}$, as in Sec. II. Notice that both sets of constraints are obtained by taking two local basis $\left\{\omega_{i}\right\}$ and $\left\{\bar{\omega}_{i}\right\}$ of the codistribution $M^{\circ}$ on $V$ and $\bar{V}$, respectively.

Then, for each one, we have the extended Lagrangians

$$
\begin{aligned}
& \mathcal{L}: T\left(V \times \mathbb{R}^{m}\right) \rightarrow \mathbb{R}, \quad \mathcal{L}=L+\lambda^{i} \mu_{i A} \dot{q}^{A}, \\
& \overline{\mathcal{L}}: T\left(\bar{V} \times \mathbb{R}^{m}\right) \rightarrow \mathbb{R}, \quad \overline{\mathcal{L}}=L+\lambda^{i} \bar{\mu}_{i A} \dot{\bar{q}}^{A},
\end{aligned}
$$

and we can apply the constraint algorithm. In this way, we obtain the constraint submanifolds $P_{2}$ and $\bar{P}_{2}$,

$$
P_{2} \equiv(T V \cap M) \times T \mathbb{R}^{m} \hookrightarrow P_{1} \equiv T\left(V \times \mathbb{R}^{m}\right),
$$




$$
\bar{P}_{2} \equiv(T \bar{V} \cap M) \times T \mathrm{R}^{m} \hookrightarrow \bar{P}_{1} \equiv T\left(\bar{V} \times \mathrm{R}^{m}\right) .
$$

Assume now that

$$
\omega_{i}=\mu_{i A}(q) d q^{A}, \quad \bar{\omega}_{i}=\bar{\mu}_{i A}(\bar{q}) d \bar{q}^{A} .
$$

Then, there exist differentiable functions

$$
\begin{aligned}
& \Lambda_{i}^{j}: V \cap \bar{V} \rightarrow \mathbb{R}^{2 m}, \\
& \bar{\Lambda}_{j}^{k}: V \cap \bar{V} \rightarrow \mathbb{R}^{2 m},
\end{aligned}
$$

which give the matrices of the change of basis at each point in $V \cap \bar{V}$,

$$
\Lambda_{i}^{j} \omega_{j}=\bar{\omega}_{i}, \quad \bar{\Lambda}_{j}^{k} \bar{\omega}_{k}=\omega_{j}, \quad \Lambda_{i}^{j} \bar{\Lambda}_{j}^{k}=\delta_{i}^{k} .
$$

Consequently, we have

$$
\begin{aligned}
\Lambda_{i}^{j} \mu_{j A} & =\bar{\mu}_{i B} \frac{\partial \bar{q}^{B}}{\partial q^{A}}, \\
\bar{\Lambda}_{j}^{k} \bar{\mu}_{k A} & =\mu_{j B} \frac{\partial q^{B}}{\partial \bar{q}^{A}} .
\end{aligned}
$$

As a first result we deduce that

$$
\bar{\Phi}_{i}=\Lambda_{i}^{j} \Phi_{j}
$$

Therefore, $P_{2} \cup \bar{P}_{2}$ can be glued to form a new submanifold of $P_{1} \cup \bar{P}_{1}$, which is in turn a submanifold of $T\left(Q \times \mathbb{R}^{m}\right)$.

Remark VIII.1: In spite of this, there is no way to extend $\mathcal{L}$ or $\overline{\mathcal{L}}$ to the whole of $P_{1} \cup \bar{P}_{1}$, so we will have to consider the process for each neighborhood.

Next, define the transformation

$$
\begin{aligned}
\bar{\Lambda}: & P_{1} \cap \bar{P}_{1} \rightarrow P_{1} \cap \bar{P}_{1} \\
\left(q^{A}, \lambda^{i}, \dot{q}^{A}, \dot{\lambda}^{i}\right) & \mapsto\left(\bar{q}^{A}, \bar{\Lambda}_{i}^{j} \lambda^{i}, \dot{\bar{q}}^{A}, \bar{\Lambda}_{i}^{j} \dot{\lambda}^{i}\right),
\end{aligned}
$$

which permits us to relate the extended Lagrangians as

$$
\overline{\mathcal{L}}_{\mid P_{1} \cap \bar{P}_{1}}{ }^{\circ} \bar{\Lambda}=L+\bar{\Lambda}_{i}^{j} \lambda^{i} \bar{\Phi}_{j}=L+\lambda^{i} \Phi_{i}=\mathcal{L}_{\mid P_{1} \cap \bar{P}_{1}} .
$$

This implies that on $P_{1} \cap \bar{P}_{1}$ we have

$$
S^{*}\left(\bar{\Lambda}^{*} d \overline{\mathcal{L}}\right)=S^{*}\left(d\left(\bar{\Lambda}^{*} \overline{\mathcal{L}}\right)\right)=S^{*}(d \mathcal{L}),
$$

and therefore the Poincaré-Cartan two-forms verify

$$
\omega_{\mathcal{L}}=\bar{\Lambda}^{*}\left(\omega_{\overline{\mathcal{L}}}\right),
$$

on $P_{1} \cap \bar{P}_{1}$. Since the energy associated with both extensions is the same, $E_{L}$, we deduce that if $\Gamma_{\mathcal{D}}$ is a solution on $P_{2}$ for the constrained system defined by $\mathcal{L}$, then $\bar{\Lambda}_{*}\left(\Gamma_{\mathcal{D}}\right)$ is a solution for the constrained system defined by $\overline{\mathcal{L}}$. In other words, if $\Gamma_{\mathcal{D}}$ satifies the equation 


$$
\left(i_{\Gamma_{\mathcal{D}}} \omega_{\mathcal{L}}=d E_{\mathcal{L}}\right)_{\mid P_{2}}
$$

then we will have

$$
\left(i_{\bar{\Lambda}_{*} \Gamma_{\mathcal{D}}} \omega_{\overline{\mathcal{L}}}=d E_{\overline{\mathcal{L}}}\right) \mid \bar{P}_{2}
$$

In terms of their integral curves, we have that an integral curve of a fixed vector field $\Gamma_{\mathcal{D}_{0}^{i}}$ of the family of solutions $\Gamma_{\mathcal{D}}$ is transformed by $\bar{\Lambda}$ into an integral curve of $\bar{\Gamma}_{\overline{\mathcal{D}}_{0}^{j}}$ on $P_{2} \cap \bar{P}_{2}$, where $\overline{\mathcal{D}}_{0}^{j} \circ \bar{\Lambda}=\bar{\Lambda}_{i}^{j} \mathcal{D}_{0}^{i}+\dot{q}^{A} \dot{\lambda}^{i}\left(\partial \bar{\Lambda}_{i}^{j} / \partial q^{A}\right)$.

Indeed, if

$$
\gamma(t)=\left(\gamma^{A}(t), \gamma^{i}(t), \widetilde{\gamma}^{A}(t), \widetilde{\gamma}^{i}(t)\right)
$$

is an integral curve of $\Gamma_{\mathcal{D}_{0}^{i}}$ on $P_{2} \cap \bar{P}_{2}$, then

$$
\bar{\gamma}(t)=\left(\gamma^{A}(t) \frac{\partial \bar{q}^{B}}{\partial q^{A}}, \gamma^{i}(t) \bar{\Lambda}_{i}^{j}(t), \widetilde{\gamma}^{A}(t) \frac{\partial \bar{q}^{B}}{\partial q^{A}}, \widetilde{\gamma}^{i}(t) \bar{\Lambda}_{i}^{j}(t)\right),
$$

will be an integral curve of $\bar{\Gamma}_{\overline{\mathcal{D}}_{0}^{j}}$ on $P_{2} \cap \bar{P}_{2}$. It is very important to observe that, although different, the projections of $\gamma(t)$ and $\bar{\gamma}(t)$ to $M$ coincide.

Remark VIII.2: If $\mathcal{S}$ (respectively, $\overline{\mathcal{S}}$ ) denotes as above the submanifold of $P_{2}$ (respectively, $\bar{P}_{2}$ ) where a SODE solution $\widetilde{\Gamma}$ (respectively, $\overline{\tilde{\Gamma}}$ ) exists, then

$$
\bar{\Lambda}_{*} \widetilde{\Gamma}=\overline{\tilde{\Gamma}} \circ \bar{\Lambda}
$$

holds on points in $\mathcal{S} \cap \overline{\mathcal{S}}$, that is, $\widetilde{\Gamma}$ and $\overline{\widetilde{\Gamma}}$ are $\bar{\Lambda}$ related on the overlapping. This can be seen as

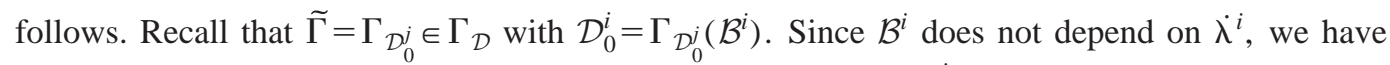
that $\Gamma_{\mathcal{D}_{0}^{j}}\left(\mathcal{B}^{i}\right)=\Gamma_{\mathcal{D}^{j}}\left(\mathcal{B}^{i}\right)$ for all $\Gamma_{\mathcal{D}^{j}} \in \Gamma_{\mathcal{D}}$ and we can compute $\mathcal{D}_{0}^{i}$ choosing any member of the family $\Gamma_{\mathcal{D}}$. The same is true for the family $\bar{\Gamma}_{\overline{\mathcal{D}}}$. Then, taking $\Gamma_{\mathcal{D}^{j}}$ and $\bar{\Gamma}_{\overline{\mathcal{D}}^{k}}$ such that $\bar{\Lambda}_{*} \Gamma_{\mathcal{D}^{j}}$ $=\bar{\Gamma}_{\overline{\mathcal{D}}^{k}} \bar{\Lambda}$, we can check that

$$
\overline{\mathcal{D}}_{0}^{i}=\bar{\Gamma}_{\overline{\mathcal{D}}^{k}}\left(\overline{\mathcal{B}}^{i}\right)=\bar{\Gamma}_{\overline{\mathcal{D}}^{k}}\left(\dot{\lambda}^{i}\right)=\Gamma_{\mathcal{D}^{j}}\left(\bar{\Lambda}_{j}^{i} \dot{\lambda}^{j}\right)=\bar{\Lambda}_{j}^{i} \mathcal{D}_{0}^{j}+\dot{q}^{A} \dot{\lambda}^{j} \frac{\partial \bar{\Lambda}_{j}^{i}}{\partial q^{A}},
$$

or, in other words, Eq. (19) holds.

Remark VIII.3: Given a "vakonomic motion," $\widetilde{c}(t)=\left(q^{A}(t)\right)$, there are different curves in $P_{2} \cap \bar{P}_{2}$ that project to $(\widetilde{c}(t), \dot{\widetilde{c}}(t)) \in M$. Indeed, if we take $\left(q_{0}^{A}, \dot{q}_{0}^{A}\right) \in M \cap T V \cap T \bar{V}$ and $\left(\lambda_{0}^{i}, \dot{\lambda}_{0}^{i}\right)$ as initial conditions for the Lagrange multipliers, we can consider the integral curve of $\widetilde{\Gamma}$ starting from $\left(q_{0}^{A}, \lambda_{0}^{i}, \dot{q}_{0}^{A}, \dot{\lambda}_{0}^{i}\right)$. Now, the curve $\bar{\gamma}=\bar{\Lambda} \circ \gamma$ will be an integral curve of $\overline{\tilde{\Gamma}}$ starting from $\left(q_{0}^{A}, \bar{\Lambda}_{i}^{j}\left(q_{0}^{A}\right) \lambda_{0}^{i}, \dot{q}_{0}^{A}, \bar{\Lambda}_{i}^{j}\left(q_{0}^{A}\right) \dot{\lambda}_{0}^{i}\right)$. Both curves project to the same solution of the vakonomic equations of motion. Therefore, in order to determine an unique curve on $M \times T \mathbb{R}^{m}$ whose projection is $(\widetilde{c}(t), \dot{\widetilde{c}}(t))$, we are forced to specify not only the initial conditions for the Lagrange multipliers, but also the set of constraint functions such that $\left(q_{0}^{A}, \lambda_{0}^{i}, \dot{q}_{0}^{A}, \dot{\lambda}_{0}^{i}\right) \in P_{2}$.

We have seen what happens in the Lagrangian formalism when changing constraint functions. Next, we accomplish the same task in the Hamiltonian context. As a consequence, we will give later a relation of the above-mentioned integral curves with the solutions of vakonomic equations of motion. By the Legendre transformations $\mathcal{F} \mathcal{L}$ and $\mathcal{F} \overline{\mathcal{L}}$ associated to $\mathcal{L}$ and $\overline{\mathcal{L}}$, respectively, we obtain the presymplectic systems $\left(M_{1}, \omega_{1}, h_{1}\right)$ and $\left(\bar{M}_{1}, \bar{\omega}_{1}, \bar{h}_{1}\right)$, where 


$$
\begin{aligned}
& M_{1}=\mathcal{F} \mathcal{L}\left(P_{1}\right), \quad \omega_{1}=j_{1}^{*}(\omega), \quad h_{1} \circ \mathcal{F} \mathcal{L}=E_{\mathcal{L}}, \quad h_{1}=\frac{1}{2} g^{A B}\left(\hat{p}_{A}-\lambda^{i} \mu_{i A}\right)\left(\hat{p}_{B}-\lambda^{j} \mu_{j B}\right)+U, \\
& \bar{M}_{1}=\mathcal{F} \overline{\mathcal{L}}\left(\bar{P}_{1}\right), \quad \bar{\omega}_{1}=\bar{J}_{1}^{*}(\omega), \quad \bar{h}_{1} \circ \mathcal{F} \overline{\mathcal{L}}=E_{\overline{\mathcal{L}}}, \quad \bar{h}_{1}=\frac{1}{2} \bar{g}^{A B}\left(\hat{\bar{p}}_{A}-\lambda^{i} \bar{\mu}_{i A}\right)\left(\hat{\bar{p}}_{B}-\lambda^{j} \bar{\mu}_{j B}\right)+U,
\end{aligned}
$$

with the obvious notations.

Notice that $M_{1} \cup \bar{M}_{1}$ can be provided of a differentiable structure such that it is a submanifold of $T^{*}(V \cup \bar{V}) \times \mathbb{R}^{m}$. We also have that the restriction of the standard symplectic form of $T^{*}(Q$ $\left.\times \mathbb{R}^{m}\right)$ to $M_{1} \cup \bar{M}_{1}$ is the natural extension of the two-forms $\omega_{1}, \bar{\omega}_{1}$. However, there is no canonical extension to $M_{1} \cup \bar{M}_{1}$ of the projected Hamiltonians $h_{1}$ and $\bar{h}_{1}$.

Define the transformations

$$
\begin{gathered}
\bar{\Lambda}: \quad M_{1} \cap \bar{M}_{1} \rightarrow M_{1} \cap \bar{M}_{1} \\
\left(q^{A}, \lambda^{i}, \hat{p}_{A}, 0\right) \mapsto\left(\bar{q}^{A}, \bar{\Lambda}_{i}^{j} \lambda^{i}, \hat{p}_{B} \frac{\partial q^{B}}{\partial \bar{q}^{A}}, 0\right),
\end{gathered}
$$

such that the following diagram is commutative:

$$
\begin{aligned}
& P_{1} \cap \bar{P}_{1} \underset{\mathcal{F L}}{\rightarrow} M_{1} \cap \bar{M}_{1} \\
& \bar{\Lambda} \downarrow \quad \downarrow \bar{\Lambda} \\
& \\
& P_{1} \cap \bar{P}_{1} \rightarrow M_{1} \cap \bar{M}_{1}
\end{aligned}
$$

We have

$$
\bar{\Lambda}^{*}\left(\bar{\omega}_{1}\right)=\omega_{1}, \quad \bar{h}_{1} \circ \bar{\Lambda}=h_{1} .
$$

Applying the algorithm to both presymplectic systems, we obtain the secondary constraint submanifolds

$$
\begin{array}{ll}
M_{2}=\left\{y \in M_{1} / \chi_{i}(y)=0\right\}, & \chi_{i}=\mu_{i A} g^{A B}\left(\hat{p}_{B}-\lambda^{j} \mu_{j B}\right), \\
\bar{M}_{2}=\left\{y \in \bar{M}_{1} / \bar{\chi}_{j}(y)=0\right\}, & \bar{\chi}_{j}=\bar{\mu}_{j A} \bar{g}^{A B}\left(\hat{\bar{p}}_{B}-\lambda^{k} \bar{\mu}_{k B}\right) .
\end{array}
$$

Observe that

$$
\chi_{i}(y)=-\left(\frac{\partial h_{1}}{\partial \lambda^{i}}\right)_{y}=-\left(\frac{\partial\left(\bar{h}_{1} \circ \bar{\Lambda}\right)}{\partial \lambda_{i}}\right)_{y}=-\bar{\Lambda}_{i}^{k}(y)\left(\frac{\partial \bar{h}_{1}}{\partial \lambda^{k}}\right)_{\bar{\Lambda}(y)}=\bar{\Lambda}_{i}^{k}\left(\bar{\chi}_{k}(\bar{\Lambda}(y)),\right.
$$

that is,

$$
\Lambda_{j}^{i} \chi_{i}=\bar{\chi}_{j} \circ \bar{\Lambda}
$$

As a consequence, the set $M_{2} \cup \bar{M}_{2}$ does not define in general a submanifold of $M_{1} \cup \bar{M}_{1} \subseteq T^{*}\left((V \cup \bar{V}) \times \mathbb{R}^{m}\right)$. However, we have a nice relation between both submanifolds, indeed,

$$
\bar{\Lambda}\left(M_{2} \cap \bar{M}_{1}\right)=\bar{M}_{2} \cap M_{1} .
$$


It is important to observe that $M_{2} \cap \bar{M}_{1}$ is an open submanifold of $M_{2}$. Therefore, on restricting the symplectic form $\omega_{2}$ to $M_{2} \cap \bar{M}_{1}$, we do not lose its symplectic character.

Remark VIII.4: A careful computation shows that $\bar{\Lambda}_{\mid M_{2} \cap \bar{M}_{2}}$ is just the identity. Consequently, we have, for example, that

$$
\left(\bar{h}_{2}\right)_{\mid M_{2} \cap \bar{M}_{2}}=\left(h_{2}\right)_{\mid M_{2} \cap \bar{M}_{2}} .
$$

In addition, using (20) and the relations:

$$
\mathcal{F} \mathcal{L}\left(\Gamma_{\mathcal{D}}\right)=\bar{\Gamma}_{M_{2}}, \quad \mathcal{F} \overline{\mathcal{L}}\left(\bar{\Gamma}_{\overline{\mathcal{D}}}\right)=\bar{\Gamma}_{\bar{M}_{2}}, \quad \bar{\Lambda}_{*} \Gamma_{\mathcal{D}}=\bar{\Gamma}_{\overline{\mathcal{D}}}^{\circ} \bar{\Lambda}
$$

we deduce that the vector fields $\bar{\Gamma}_{M_{2}}$ and $\bar{\Gamma}_{\bar{M}_{2}}$ fulfill along $M_{2} \cap \bar{M}_{1}$

$$
\bar{\Lambda}_{*} \bar{\Gamma}_{M_{2}}=\bar{\Gamma}_{\bar{M}_{2}} \circ \bar{\Lambda}
$$

We see that the integral curves of $\Gamma_{M_{2}}$ and $\bar{\Gamma}_{\bar{M}_{2}}$ on $M_{2} \cap \bar{M}_{2}$ are, in principle, different. However, one can easily check that their projections onto $\bar{M}$ by

$$
\begin{aligned}
& \tilde{\pi}: M_{2} \rightarrow \bar{M}, \quad\left(q^{A}, \lambda^{i}, \hat{p}_{A}, 0\right) \mapsto\left(q^{A}, \hat{p}_{A}-\lambda^{i} \mu_{i A}\right), \\
& \tilde{\bar{\pi}}: \bar{M}_{2} \rightarrow \bar{M}, \quad\left(\bar{q}^{A}, \lambda^{i}, \hat{\bar{p}}_{A}, 0\right) \mapsto\left(\bar{q}^{A}, \hat{\bar{p}}_{A}-\lambda^{i} \bar{\mu}_{i A}\right),
\end{aligned}
$$

coincide, since

$$
\tilde{\bar{\pi}} \circ \bar{\Lambda}_{\mid M_{2} \cap \bar{M}_{1}}=\widetilde{\pi}_{\mid M_{2} \cap \bar{M}_{1}} \cdot
$$

We will now investigate the relation between the corresponding Dirac brackets, and more interesting, about the induced brackets on the final constraint submanifolds $M_{2}$ and $\bar{M}_{2}$,

$$
\begin{aligned}
& \{,\}_{\mathcal{D} \mid M_{2}}=\left(\{,\}-\left\{, \varphi_{\alpha}\right\} C^{\alpha \beta}\left\{\varphi_{\beta},\right\}\right)_{\mid M_{2}}, \\
& \{,\}_{\mathcal{D} \mid \bar{M}_{2}}=\left(\{,\}-\left\{, \bar{\varphi}_{\alpha}\right\} \bar{C}^{\alpha \beta}\left\{\bar{\varphi}_{\beta},\right\}\right)_{\mid \bar{M}_{2}} .
\end{aligned}
$$

Recall that $\bar{\Lambda}^{*}\left(\bar{\omega}_{1}\right)_{\mid M_{1} \cap \bar{M}_{1}}=\left(\omega_{1}\right)_{\mid M_{1} \cap \bar{M}_{1}}$. This fact implies that $\bar{\Lambda} *\left(\bar{\omega}_{2}\right)_{\mid \bar{M}_{2} \cap M_{1}}=\left(\omega_{2}\right)_{\mid M_{2} \cap \bar{M}_{1}}$. Consequently, we have for each pair of functions, $f, g: \bar{M}_{2} \rightarrow \mathbb{R}$ that

$$
\{f, g\}^{*} \circ \bar{\Lambda}_{\mid M_{2} \cap \bar{M}_{1}}=\{\tilde{f}, \widetilde{g}\}^{*} \circ k,
$$

where $k: M_{2} \cap \bar{M}_{1} \hookrightarrow M_{2}$ is the canonical inclusion and $\tilde{f}, \widetilde{g}: M_{2} \rightarrow \mathbb{R}$ are extensions to $M_{2}$ of $\bar{\Lambda}_{\mid M_{2} \cap \bar{M}_{1}}{ }^{\circ} f_{\mid \bar{M}_{2} \cap M_{1}}, \bar{\Lambda}_{\mid M_{2} \cap \bar{M}_{1}}{ }^{\circ} g_{\mid \bar{M}_{2} \cap M_{1}}$, respectively.

As a consequence, when defining the vakonomic brackets for functions $f, g$ on $\bar{M}$ we have the following two possibilities:

$$
\begin{aligned}
& \{f, g\}_{\mathrm{vak}}=\{f \circ \widetilde{\pi}, g \circ \widetilde{\pi}\}_{M_{2}}, \\
& \{f, g\}_{\overline{\mathrm{vak}}}=\{f \circ \widetilde{\bar{\pi}}, g \circ \widetilde{\bar{\pi}}\}_{\bar{M}_{2}} .
\end{aligned}
$$

However, the relation $\widetilde{\bar{\pi}} \circ \bar{\Lambda}_{\mid M_{2} \cap \bar{M}_{1}}=\widetilde{\pi}_{\mid M_{2} \cap \bar{M}_{1}}$ and (22) imply that 


$$
\{f, g\}_{\mathrm{vak}^{\circ}}{ }^{\circ}=\{f \circ \widetilde{\pi}, g \circ \widetilde{\pi}\}_{M_{2}} \circ k=\left\{f \circ \tilde{\bar{\pi}}, g \circ \tilde{\bar{\pi}}_{\bar{M}_{2}}{ }^{\circ} \bar{\Lambda}_{\mid M_{2} \cap \bar{M}_{1}}=\{f, g\} \overline{\mathrm{vak}}^{\circ} \bar{\Lambda}_{\mid M_{2} \cap \bar{M}_{1}},\right.
$$

which is coherent with the above-mentioned formula $\bar{\Lambda}_{*} \bar{\Gamma}_{M_{2}}=\bar{\Gamma}_{\bar{M}_{2}} \circ \bar{\Lambda}$.

Remark VIII.5: Therefore, although different, both brackets give the same valid information about the evolution of a dynamical variable along "vakonomic curves" on $\bar{M}$. In fact, given a "vakonomic" curve on $\bar{M}, \bar{c}(t)=\left(q^{A}(t), p_{A}(t)\right)$, we take $\gamma(t)=\left(q^{A}(t), \lambda^{i}(t), \hat{p}_{A}(t), 0\right)$ on $M_{2} \cap \bar{M}_{1}$ and $\bar{\Lambda} \circ \gamma(t)=\left(\bar{q}^{A}(t), \bar{\Lambda}_{i}^{j} \lambda^{i}(t), \hat{\bar{p}}_{A}(t), 0\right)$ on $\bar{M}_{2} \cap M_{1}$ projecting onto it. Then, the evolution of $f$ onto this curve on $\bar{M}$ will be

$$
\frac{d}{d t}\left(f\left(q^{A}(t), p_{A}(t)\right)\right)=\frac{d}{d t}\left(f \circ \tilde{\widetilde{\pi}}\left(\bar{q}^{A}(t), \bar{\Lambda}_{i}^{j} \lambda^{i}(t), \hat{\bar{p}}_{A}(t), 0\right)\right)=\frac{d}{d t}\left(f \circ \widetilde{\pi}\left(q^{A}(t), \lambda^{i}(t), \hat{p}_{A}(t), 0\right)\right),
$$

that is,

$$
\bar{f}_{\mid \bar{c}} \equiv \bar{\Gamma}_{\mid \bar{M}_{2}}(f \circ \tilde{\bar{\pi}})_{\mid \bar{\Lambda} \circ \gamma}=\bar{\Gamma}_{M_{2}}(f \circ \widetilde{\pi})_{\mid \gamma} \equiv \dot{f}_{\mid \bar{c}},
$$

or, equivalently,

$$
\bar{f} \equiv\left\{f, H_{\mid \bar{M}}\right\}_{\overline{\mathrm{vak}}} \bar{\Lambda}=\left\{f, H_{\mid \bar{M}}\right\}_{\mathrm{vak}} \equiv \dot{f} .
$$

Example VIII.6: The vakonomic particle. We consider the case of a particle of unit mass moving through the space $Q=\mathbb{R}^{3}$ subjected to the global nonholonomic constraint $\Phi=\dot{z}-y \dot{x}$. In order to illustrate the precedent discussion, we will take, instead of $\Phi$, the following constraints:

$$
\begin{gathered}
\phi: T U \rightarrow \mathbb{R}, \phi(x, y, z, \dot{x}, \dot{y}, \dot{z})=x(\dot{z}-y \dot{x}), \\
\psi: T V \rightarrow \mathbb{R}, \psi(x, y, z, \dot{x}, \dot{y}, \dot{z})=z(\dot{z}-y \dot{x}),
\end{gathered}
$$

where

$$
\begin{aligned}
& U=\left\{(x, y, z) \in \mathbb{R}^{3} / x \neq 0\right\}, \\
& V=\left\{(x, y, z) \in \mathbb{R}^{3} / z \neq 0\right\} .
\end{aligned}
$$
are

Here, the Lagrangian $L$ is the kinetic energy $L=\frac{1}{2}\left(\dot{x}^{2}+\dot{y}^{2}+\dot{z}^{2}\right)$, so the extended Lagrangians

$$
\begin{array}{cc}
\mathcal{L}_{\phi}: T(U \times \mathbb{R}) \rightarrow \mathbb{R}, & \mathcal{L}_{\phi}=\frac{1}{2}\left(\dot{x}^{2}+\dot{y}^{2}+\dot{z}^{2}\right)+\lambda(x \dot{z}-x y \dot{x}), \\
\mathcal{L}_{\psi}: T(V \times \mathbb{R}) \rightarrow \mathbb{R}, & \mathcal{L}_{\psi}=\frac{1}{2}\left(\dot{x}^{2}+\dot{y}^{2}+\dot{z}^{2}\right)+\lambda(z \dot{z}-z y \dot{x}) .
\end{array}
$$

Since $(x / z) \psi=\phi$ in $T U \cap T V$, the transformation $\bar{\Lambda}$ is given by

$$
\begin{aligned}
& \bar{\Lambda}: \quad T((U \cap V) \times \mathbb{R}) \quad \rightarrow \quad T((U \cap V) \times \mathbb{R}) \\
& (x, y, z, \lambda, \dot{x}, \dot{y}, \dot{z}, \dot{\lambda}) \mapsto\left(x, y, z, \frac{x}{z} \lambda, \dot{x}, \dot{y}, \dot{z}, \frac{x}{z} \dot{\lambda}\right) .
\end{aligned}
$$

The two-forms of Poincaré-Cartan are, respectively,

$$
\begin{aligned}
& \omega_{\mathcal{L}_{\phi}}=d x \wedge d \dot{x}-x y d x \wedge d \lambda-\lambda x d x \wedge d y+d y \wedge d \dot{y}+d z \wedge d \dot{z}+\lambda d z \wedge d x+x d z \wedge d \lambda, \\
& \omega_{\mathcal{L}_{\psi}}=d x \wedge d \dot{x}-z y d x \wedge d \lambda-\lambda z d x \wedge d y-\lambda y d x \wedge d z+d y \wedge d \dot{y}+d z \wedge d \dot{z}+z d z \wedge d \lambda .
\end{aligned}
$$


Let $\Gamma_{\phi}, \Gamma_{\psi}$ be the vector fields on $P_{2}^{\phi}, P_{2}^{\psi}$ satisfying

$$
i_{\Gamma_{\phi}} \omega_{\mathcal{L}_{\phi}}=d E_{L}, \quad i_{\Gamma_{\psi}} \omega_{\mathcal{L}_{\psi}}=d E_{L}
$$

Then, the coefficients must fulfill the following equations:

$$
\begin{gathered}
\mathcal{C}_{x}^{\phi}=\left(\lambda \dot{y}+y \mathcal{B}_{\lambda}^{\phi}\right) x+\lambda \dot{z}, \quad \mathcal{C}_{x}^{\psi}=\left(\lambda \dot{y}+y \mathcal{B}_{\lambda}^{\psi}\right) z+\lambda y \dot{z}, \\
\mathcal{C}_{y}^{\phi}=-\lambda x \dot{x}, \quad \mathcal{C}_{y}^{\psi}=-\lambda z \dot{x}, \\
\mathcal{C}_{x}^{\phi}=-\lambda \dot{x}-\mathcal{B}_{\lambda}^{\phi} x, \quad \mathcal{C}_{z}^{\psi}=-\lambda y \dot{x}-\mathcal{B}_{\lambda}^{\psi} z .
\end{gathered}
$$

The tangency conditions $\Gamma_{\phi}(\phi)=0, \Gamma_{\psi}(\psi)=0$ are reduced to

$$
\mathcal{C}_{z}^{\phi}-\dot{y} \dot{x}-y \mathcal{C}_{x}^{\phi}=0, \quad \mathcal{C}_{z}^{\psi}-\dot{y} \dot{x}-y \mathcal{C}_{x}^{\psi}=0 .
$$

It is easy to see now that in each case we obtain

$$
\begin{aligned}
& \mathcal{B}_{\lambda}^{\phi}=-\lambda \frac{\dot{x}}{x}-\frac{\dot{y}(\dot{x}+\lambda x y)}{x\left(1+y^{2}\right)}, \\
& \mathcal{B}_{\lambda}^{\psi}=-\lambda \frac{\dot{z}}{z}-\frac{\dot{y}(\dot{x}+\lambda z y)}{z\left(1+y^{2}\right)},
\end{aligned}
$$

so that we have

$$
\begin{gathered}
\mathcal{C}_{x}^{\phi}=\lambda x \dot{y}-\frac{y \dot{y}(\dot{x}+\lambda x y)}{1+y^{2}}, \quad \mathcal{C}_{x}^{\psi}=\lambda y \dot{z}-\frac{y \dot{y}(\dot{x}+\lambda z y)}{1+y^{2}}, \\
\mathcal{C}_{y}^{\phi}=-\lambda x \dot{x}, \quad \mathcal{C}_{y}^{\psi}=-\lambda z \dot{x}, \\
\mathcal{C}_{z}^{\phi}=\frac{\dot{y}(\dot{x}+\lambda x y)}{1+y^{2}}, \quad \mathcal{C}_{z}^{\psi}=\frac{\dot{y}(\dot{x}+\lambda z y)}{1+y^{2}} .
\end{gathered}
$$

Consequently, we have determined the families $\Gamma_{\mathcal{D}}^{\phi}$ and $\Gamma_{\mathcal{D}}^{\psi}$. If we denote by $\mathcal{S}^{\phi}, \mathcal{S}^{\psi}$ the submanifolds of $P_{2}^{\phi}, P_{2}^{\psi}$, respectively,

$$
\begin{aligned}
& \mathcal{S}^{\phi}=\left\{y \in T\left(U \times \mathbb{R}^{3}\right) / \dot{\lambda}=-\lambda \frac{\dot{x}}{x}-\frac{\dot{y}(\dot{x}+\lambda x y)}{x\left(1+y^{2}\right)}\right\}, \\
& \mathcal{S}^{\psi}=\left\{y \in T\left(V \times \mathbb{R}^{3}\right) / \dot{\lambda}=-\lambda \frac{\dot{z}}{z}-\frac{\dot{y}(\dot{x}+\lambda z y)}{z\left(1+y^{2}\right)}\right\},
\end{aligned}
$$

we have proved that there is a vector field $\widetilde{\Gamma}_{\phi}$ (respectively, $\widetilde{\Gamma}_{\psi}$ ) of $\Gamma_{\mathcal{D}}^{\phi}\left(\right.$ respectively, $\left.\Gamma_{\mathcal{D}}^{\psi}\right)$ satisfying the SODE condition and tangent to $\mathcal{S}^{\phi}$ (respectively, $\mathcal{S}^{\psi}$ ). These vector fields are determined by

$$
\begin{aligned}
\mathcal{D}_{\lambda}^{\phi}= & -\dot{\lambda}\left(\frac{\dot{x}}{x}+\frac{y \dot{y}}{1+y^{2}}\right)+\left(\frac{\dot{x}}{x}\right)^{2}\left(\lambda+\frac{\dot{y}}{1+y^{2}}\right)+\frac{2 y \dot{x} \dot{y}^{2}}{x\left(1+y^{2}\right)^{2}} \\
& +\lambda \frac{\dot{y}^{2}\left(y^{2}-1\right)}{\left(1+y^{2}\right)^{2}}-\mathcal{C}_{x}^{\phi}\left[\frac{\dot{y}}{x\left(1+y^{2}\right)}+\frac{\lambda}{x}\right]-\mathcal{C}_{y}^{\phi}\left[\frac{\dot{x}}{x\left(1+y^{2}\right)}+\frac{\lambda y}{1+y^{2}}\right],
\end{aligned}
$$




$$
\begin{aligned}
\mathcal{D}_{\lambda}^{\psi}= & -\dot{\lambda}\left(\frac{\dot{z}}{z}+\frac{y \dot{y}}{1+y^{2}}\right)+\left(\frac{\dot{z}}{z}\right)^{2}\left(\lambda+\frac{\dot{y}}{y\left(1+y^{2}\right)}\right)+\frac{2 y \dot{y}^{2}(\dot{x}+\lambda z y)}{\left(1+y^{2}\right)^{2}} \\
& -\lambda \frac{\dot{y}^{2}}{\left(1+y^{2}\right)^{2}}-\frac{\lambda}{z} \mathcal{C}_{z}^{\psi}-\mathcal{C}_{x}^{\psi} \frac{\dot{y}}{z\left(1+y^{2}\right)}-\mathcal{C}_{y}^{\psi}\left[\frac{\dot{x}}{z\left(1+y^{2}\right)}+\frac{\lambda y}{1+y^{2}}\right] .
\end{aligned}
$$

A straightforward but tedious computation shows that

$$
\bar{\Lambda}_{*} \widetilde{\Gamma}_{\phi}=\widetilde{\Gamma}_{\psi} \circ \bar{\Lambda}
$$
are

We pass now to the Hamiltonian description of the problem. The Legendre transformations

$$
\begin{aligned}
& \mathcal{F L}_{\phi}: \quad T(U \times \mathbb{R}) \quad \longrightarrow \quad T^{*}(U \times \mathbb{R}) \\
& (x, y, z, \lambda, \dot{x}, \dot{y}, \dot{z}, \dot{\lambda}) \quad \mapsto \quad(x, y, z, \lambda, \dot{x}-\lambda x y, \dot{y}, \dot{z}+\lambda x, 0), \\
& \mathcal{F L}_{\psi}: \quad T(V \times \mathbb{R}) \quad \longrightarrow \quad T^{*}(V \times \mathbb{R}) \\
& (x, y, z, \lambda, \dot{x}, \dot{y}, \dot{z}, \dot{\lambda}) \quad \mapsto \quad(x, y, z, \lambda, \dot{x}-\lambda z y, \dot{y}, \dot{z}+\lambda z, 0) .
\end{aligned}
$$

Therefore, we have that

$$
\begin{aligned}
& M_{1}^{\phi}=\mathcal{F} \mathcal{L}_{\phi}(T(U \times \mathbb{R}))=\left\{x \neq 0, \hat{p}_{\lambda}=0\right\} \equiv \mathbb{R}^{7} /\{x=0\}, \\
& M_{1}^{\psi}=\mathcal{F} \mathcal{L}_{\psi}(T(V \times \mathbb{R}))=\left\{z \neq 0, \hat{p}_{\lambda}=0\right\} \equiv \mathbb{R}^{7} /\{z=0\},
\end{aligned}
$$

with Poincaré-Cartan two-forms and Hamiltonian functions given by

$$
\begin{gathered}
\omega_{\phi}=d x \wedge d \hat{p}_{x}+d y \wedge d \hat{p}_{y}+d z \wedge d \hat{p}_{z}, \\
h_{1}^{\phi}=\frac{1}{2}\left[\left(\hat{p}_{x}+\lambda x y\right)^{2}+\hat{p}_{y}^{2}+\left(\hat{p}_{z}-\lambda x\right)^{2}\right], \\
\omega_{\psi}=d x \wedge d \hat{p}_{x}+d y \wedge d \hat{p}_{y}+d z \wedge d \hat{p}_{z}, \\
h_{1}^{\psi=}=\frac{1}{2}\left[\left(\hat{p}_{x}+\lambda z y\right)^{2}+\hat{p}_{y}^{2}+\left(\hat{p}_{z}-\lambda z\right)^{2}\right] .
\end{gathered}
$$

It is inmediate to see that $h_{1}^{\psi} \circ \bar{\Lambda}=h_{1}^{\phi}$. The corresponding secondary constraints are

$$
\begin{aligned}
& \chi_{\phi}=-\frac{\partial h_{1}^{\phi}}{\partial \lambda}=x\left(-\left(\hat{p}_{x}+\lambda x y\right) y+\hat{p}_{z}-\lambda x\right), \\
& \chi_{\psi}=-\frac{\partial h_{1}^{\psi}}{\partial \lambda}=z\left(-\left(\hat{p}_{x}+\lambda z y\right) y+\hat{p}_{z}-\lambda z\right),
\end{aligned}
$$

and, in fact, we verify that $(z / x) \chi_{\phi}=\chi_{\psi} \circ \bar{\Lambda}$. The final constraint submanifolds in the Hamiltonian side are

$$
\begin{aligned}
& M_{2}^{\phi}=\left\{w \in M_{1}^{\phi} / \lambda=\frac{\hat{p}_{z}-y \hat{p}_{x}}{x\left(1+y^{2}\right)}\right\} \equiv \mathbb{R}^{6} /\{x=0\}, \\
& M_{2}^{\psi}=\left\{w \in M_{1}^{\psi} / \lambda=\frac{\hat{p}_{z}-y \hat{p}_{x}}{z\left(1+y^{2}\right)}\right\} \equiv \mathbb{R}^{6} /\{z=0\},
\end{aligned}
$$

with two-forms and Hamiltonians 


$$
\begin{gathered}
\omega_{\phi}=d x \wedge d \hat{p}_{x}+d y \wedge d \hat{p}_{y}+d z \wedge d \hat{p}_{z}, \\
\omega_{\psi}=d x \wedge d \hat{p}_{x}+d y \wedge d \hat{p}_{y}+d z \wedge d \hat{p}_{z}, \\
h_{2}^{\phi}=h_{2}^{\psi}=\frac{1}{2}\left(\frac{\left(\hat{p}_{x}+y \hat{p}_{z}\right)^{2}}{1+y^{2}}+\hat{p}_{y}^{2}\right) .
\end{gathered}
$$

Note that $\omega_{\phi}$ and $\omega_{\psi}$ are not the same two-form, because they are defined on different manifolds, that is, $M_{2}^{\phi}$ and $M_{2}^{\psi}$, respectively.

To define the vakonomic brackets, we have

$$
\begin{aligned}
& \tilde{\pi}_{\phi}: \quad M_{2}^{\phi} \quad \longrightarrow \quad \bar{M} \\
& \left(x, y, z, \hat{p}_{x}, \hat{p}_{y}, \hat{p}_{z}\right) \quad \mapsto \quad\left(x, y, z, \hat{p}_{x}+y \frac{\hat{p}_{z}-y \hat{p}_{x}}{1+y^{2}}, \hat{p}_{y}, \hat{p}_{z}-\frac{\hat{p}_{z}-y \hat{p}_{x}}{1+y^{2}}\right), \\
& \widetilde{\pi}_{\psi}: \quad M_{2}^{\psi} \longrightarrow \quad \bar{M} \\
& \left(x, y, z, \hat{p}_{x}, \hat{p}_{y}, \hat{p}_{z}\right) \quad \mapsto \quad\left(x, y, z, \hat{p}_{x}+y \frac{\hat{p}_{z}-y \hat{p}_{x}}{1+y^{2}}, \hat{p}_{y}, \hat{p}_{z}-\frac{\hat{p}_{z}-y \hat{p}_{x}}{1+y^{2}}\right) .
\end{aligned}
$$

Given $f, g: \bar{M} \rightarrow \mathbb{R}$, we have on $M_{2}^{\phi} \cap M_{1}^{\psi}$ that

$$
\{f, g\}_{\text {vak }}^{\phi}=\left\{f \circ \widetilde{\pi}_{\phi}, g \circ \widetilde{\pi}_{\phi}\right\}_{M_{2}^{\phi}}=\left\{f \circ \widetilde{\pi}_{\psi}, g \circ \widetilde{\pi}_{\psi\}_{M}}\right\}_{2} \bar{\Lambda}=\{f, g\}_{\text {vak }}^{\psi} \circ \bar{\Lambda} .
$$

\section{ACKNOWLEDGMENTS}

This work has been partially supported through Grant No. DGICYT (Spain) PB97-1257. S. M. and J. C. wish to thank Spanish Ministerio de Educación y Cultura for FPI and FPU grants, respectively. The authors wish to thank D. Martín de Diego for helpful comments and suggestions. We acknowledge the referee for his useful remarks.

${ }^{1}$ V. I. Arnold, Dynamical Systems (Springer, New York, 1988), Vol. III.

${ }^{2}$ J. Neimark and N. Fufaev, Dynamics of Nonholonomic Systems, Transactions of Mathematical Monographs Vol. 33 (American Mathematical Society, Providence, RI, 1972).

${ }^{3}$ E. J. Saletan and A. H. Cromer, "A variational principle for nonholonomic systems,'” Am. J. Phys. 38, 892-897 (1970).

${ }^{4}$ A. M. Vershik, Classical and Non-classical Dynamics with Constraints, Global Analysis-Studies and Applications. I [Lect. Notes Math. 1108, 218-301 (1984)].

${ }^{5}$ A. M. Vershik and L. D. Faddeev, "Differential geometry and Lagrangian mechanics with constraints," Sov. Phys. Dokl. 17, 34-36 (1972).

${ }^{6}$ V. V. Kozloz, “Realization of nonintegrable constraints in classical mechanics,” Dokl. Akad. Nauk SSSR 272, 550-554 (1983) (Russian); English transl.: Sov. Phys. Dokl. 28, 735-737 (1983).

${ }^{7}$ F. Cardin and M. Favretti, "On nonholonomic and vakonomic dynamics of mechanical systems with nonintegrable constraints," J. Geom. Phys. 18, 295-325 (1996).

${ }^{8}$ A. D. Lewis and R. M. Murray, "Variational principles for constrained systems: Theory and experiment," Int. J. Nonlinear Mech. 30, 793-815 (1995).

${ }^{9}$ L. Bates and J. Śniatycki, “Nonholonomic reduction,” Rep. Math. Phys. 32, 99-115 (1992).

${ }^{10}$ R. Cushman, D. Kemppainen, J. Śniatycki, and L. Bates, “Geometry of nonholonomic constraints,” Rep. Math. Phys. 36, 275-286 (1995).

${ }^{11}$ J. Śniatycki, "Non-holonomic Noether theorem and reduction of symmetries," in Proceedings of the Workshop on Non-Holonomic Constraints in Dynamics, Calgary, 26-29 August 1997 [Rep. Math. Phys. 42, 5-23 (1998)].

${ }^{12}$ A. M. Bloch, P. S. Krishnaprasad, J. E. Marsden, and R. M. Murray, "Nonholonomic mechanical systems with symmetry,"' Arch. Ration. Mech. Anal. 136, 21-99 (1996).

${ }^{13}$ F. Cantrijn, M. de León, J. C. Marrero, and D. Martín de Diego, "Reduction of nonholonomic mechanical systems with symmetries," in Ref. 11, pp. 25-45.

${ }^{14}$ F. Cantrijn, M. de León, J. C. Marrero, and D. Martín de Diego, “Reduction of constrained systems with symmetry," J. Math. Phys. 40, 795-820 (1999).

${ }^{15}$ J. Koiller, "Reduction of some classical non-holonomic systems with symmetry," Arch. Ration. Mech. Anal. 118, 113-148 (1992).

${ }^{16}$ M. de León, J. C. Marrero, and D. M. de Diego, “Mechanical systems with non-linear constraints,” Int. J. Theor. Phys. 36, 973-989 (1997). 
${ }^{17}$ M. de León and D. Martín de Diego, "On the geometry of non-holonomic Lagrangian systems," J. Math. Phys. 37, 3389-3414 (1996).

${ }^{18}$ C.-M. Marle, "Reduction of constrained mechanical systems and stability of relative equilibria," Commun. Math. Phys. 174, 295-318 (1995)

${ }^{19}$ C.-M. Marle, "Various approaches to conservative and nonconservative nonholonomic systems," in Ref. 11, pp. 211229.

${ }^{20}$ A. J. van der Schaft and B. M. Maschke, “On the Hamiltonian formulation of nonholonomic mechanical systems," Rep. Math. Phys. 34, 225-233 (1994)

${ }^{21}$ F. Cantrijn, M. de León, and D. Martín de Diego, "On almost-Poisson structures in nonholonomic mechanics," Nonlinearity 12, 721-737 (1999).

${ }^{22}$ W. S. Koon and J. E. Marsden, "The Hamiltonian and Lagrangian approaches to the dynamics of nonholonomic systems," Rep. Math. Phys. 40, 21-62 (1997).

${ }^{23}$ W. S. Koon and J. E. Marsden, "Poisson reduction of nonholonomic mechanical systems with symmetry," in Ref. 11, pp. 101-134.

${ }^{24}$ A. M. Bloch and P. E. Crouch, "Nonholonomic and vakonomic control systems on Riemannian manifolds," Dynamics and Control of Mechanical Systems, Waterloo, ON, 1992, Fields Institute Communication Vol. 1 (American Mathematical Society, Providence, RI, 1993), pp. 25-52.

${ }^{25}$ R. W. Brockett, "Control theory and singular Riemannian geometry," in New Directions in Applied Mathematics, edited by P. J. Hilton and G. S. Young (Springer, New York, 1982), pp. 11-27.

${ }^{26}$ M. de León and D. Martín de Diego, "Conservation laws and symmetry in economic growth models: A geometrical approach," Extracta Mathematicae 13, 335-348 (1998).

${ }^{27}$ J. Koiller and J. Delgado, "On efficiency calculations for nonholonomic locomotion problems: An application to microswimming," in Ref. 11, pp. 165-183.

${ }^{28}$ M. de León, J. C. Marrero, and D. M. de Diego, "Vakonomic mechanics versus non-holonomic mechanics: A unified geometrical approach" (unpublished).

${ }^{29}$ P. A. M. Dirac, Lectures on Quantum Mechanics (Belfer Graduate School of Science, Yeshiva University, New York, 1964).

${ }^{30}$ J. F. Cariñena and M. F. Rañada, "Comments on the presymplectic formalism and the theory of regular Lagrangians with constraints," J. Phys. A 28, L91-L97 (1995).

${ }^{31}$ M. J. Gotay, "Presymplectic manifolds, geometric constraint theory and the Dirac-Bergmann theory of constraints," Ph.D. thesis, Center for Theoretical Physics, University of Maryland, 1979.

${ }^{32}$ M. J. Gotay and J. M. Nester, "Presymplectic Lagrangian systems. I. The constraint algorithm and the equivalence theorem," Ann. Inst. Henri Poincaré, Sect. A 30, 129-142 (1979).

${ }^{33}$ M. J. Gotay and J. M. Nester, "Presymplectic Lagrangian systems. II. The second-order differential equation problem," Ann. Inst. Henri Poincaré, Sect. A 32, 1-13 (1980).

${ }^{34}$ A. Ibort, M. de León, J. C. Marrero, and D. Martín de Diego, "'Dirac brackets in constrained dynamics," Fortschr. Phys. 47, 459-492 (1999).

${ }^{35}$ L. Bates, "Examples of singular nonholonomic reduction," in Ref. 11, pp. 231-247.

${ }^{36}$ X. Gràcia, J. Marin-Solano, and M. Muñoz-Lecanda, "Variational principles in mechanics: Geometric aspects," Proceedings of the Seventh Fall Workshop, Geometry and Physics, Valencia, September 1998 (unpublished).

${ }^{37}$ M. de León and P. R. Rodrigues, Methods of Differential Geometry in Analytical Mechanics, North-Holland Mathematical Series Vol. 152 (North-Holland, Amsterdam, 1989).

${ }^{38}$ F. Cantrijn, J. F. Cariñena, M. Crampin, and L. A. Ibort, “Reduction of degenerate Lagrangian systems,' J. Geom. Phys. 3, 353-400 (1986)

${ }^{39}$ J. F. Cariñena and L. A. Ibort, "'Geometric theory of the equivalence of Lagrangians for constrained systems,' J. Phys. A 18, 3335-3341 (1985). 